\title{
Inverse heat conduction problem: Sensitivity coefficient insights, filter coefficients and intrinsic verification ${ }^{1}$
}

Keywords: Inverse heat conduction problem, sensitivity coefficients, filter coefficients

\begin{abstract}
The inverse heat conduction problem is the estimation of the time and/or space dependence of the surface heat flux or temperature utilizing interior temperature measurements at discrete times and/or locations. This problem is ill-posed since it is very sensitive to omnipresent measurement errors. Many solution methods have been proposed including exact-matching, function specification, Tikhonov regularization, iterative regularization, conjugate gradient, steepest descent and singular value decomposition. In this paper, the tools provided by the scaled sensitivity coefficients, digital filter coefficients and intrinsic verification are used to investigate and compare several of these methods. The utility of digital filters designed for on-line instrumentation for “continuous” measurements of the surface heat flux and temperature in manufacturing settings is also demonstrated.
\end{abstract}

\section{Introduction}

The inverse heat conduction problem (IHCP) involves the determination of the heat flux at the surface of a solid using one or more measured internal temperature histories. A number of methods have been proposed including function specification [1,2], Tikhonov regularization (TR) $[1,3,4,5]$, conjugate gradient (CG) $[6,7,8,9,10]$ and singular value decomposition (SVD) methods $[11,12,13,14]$. The problem is challenging because it is ill-posed which is, in part, caused by the internal measurements being delayed and damped compared to the heated surface.

This paper has two main objectives. The first is to provide a method of comparison and evaluation of some available methods for the IHCP. The comparison is accomplished by providing the digital filters $[15,16,17,18]$ underlying methods such as function specification, Tikhonov regularization and singular value decomposition methods. Reference 8 discusses Tikhonov regularization and filter coefficients but does not provide a comparison with the other two methods. The second objective is to derive some filter coefficients that can enable a near

\footnotetext{
${ }^{1}$ This paper is an extension of the plenary lecture delivered by the lead author to the 2014 International Conference on Inverse Problems in Engineering in Krakow, Poland, in May, 2014.
} 
real-time analysis; it can be helpful in real-time measurements using flame thermometers [19] and manufacturing applications. It might also have application to surface heat flux and temperature measurements in an aerospace plane or rocket re-entering the earth's atmosphere.

In order to make the task more manageable, only a single interior temperature history is considered and it is at the center of Cartesian body of thickness $L$. The location of interest is at $\tilde{x}=x / L=0.5$. Two types of boundary conditions are considered at $x=L$, homogeneous in each case. The first case is for a zero temperature rise at $x=L$ with the notation X21B?0T0 and the second case is insulated at $x=L$ and denoted X22B?0T0. Filter coefficients are found for various cases, with the emphasis on the insulated remote boundary case. Related quantities are found that provide insight and contribute to intrinsic verification.

A brief explanation of the numbering system for solutions follows. The first character is a geometric designator, and ' $\mathrm{X}$ ' denotes a Cartesian system. The next two digits are disignators for the boundary condition at the two interfaces ( $x=0$ and $x=L$ ), and the number ' 1 ' denotes a fixed temperature condition, while ' 2 ' denotes a specified heat flux condition. The letter ' $\mathrm{B}$ ' indicates that the fields following that letter are boundary condition "modifiers" that indicate the nature of the conditions at the two boundaries just specified. The ' 0 ' indicates that the heat flux at the second boundary $(x=L)$ is homogeneous. The '?' designation is introduced in this paper and supplements notation described elsewhere (see [20, pages 47-60] or [21] for more on the solution numbering system). The '?' indicates that the heat flux at the first boundary $(x=0)$ is unknown and is to be found in the solution. In fact, the '?' identifies this problem as an inverse problem. This is an important distinction from the notation described in Ref. [20] where the modifier '-' indicates an arbitrary time variation, and compound modifier ' $x$-' denotes an arbitrary functions of space.

This paper discusses sensitivity coefficients, filter formulations and optimal comparison criteria for the inverse heat conduction problem. Based on the criteria, a comparison is given of the function specification, Tikhonov regularization, conjugate gradient, and singular value decomposition methods. Two heat flux time-variation cases are considered for each of these methods and a ranking is given based on the results.

\section{Preliminary aspects}

A parallel treatment of the following discussion is given elsewhere [16, see also Ref. 1, pages 1011, 83-85, and 148-152]. The measured temperature vector $\mathbf{Y}$ which contains $N$ elements is 


$$
\mathbf{Y}=\left[\begin{array}{lllll}
Y_{1} & Y_{2} & \cdots & Y_{N-1} & Y_{N}
\end{array}\right]^{T}
$$

The true temperature vector $\mathbf{T}$ and the additive error vector $\boldsymbol{\varepsilon}$ are

$$
\begin{aligned}
\mathbf{T} & =\left[\begin{array}{lllll}
T_{1} & T_{2} & \cdots & T_{N-1} & T_{N}
\end{array}\right]^{T} \\
\boldsymbol{\varepsilon} & =\left[\begin{array}{lllll}
\varepsilon_{1} & \varepsilon_{2} & \cdots & \varepsilon_{N-1} & \varepsilon_{N}
\end{array}\right]^{T}
\end{aligned}
$$

Each of the above elements in Eqs. (1a, b, c) are at the same location; the subscripts indicate the running times. The measured temperature vector, true temperature vector and error vector are related in the additive manner

$$
\mathbf{Y}=\mathbf{T}+\boldsymbol{\varepsilon}
$$

The true, but unknown, surface heat flux vector is similarly given by

$$
\mathbf{q}=\left[\begin{array}{lllll}
q_{1} & q_{2} & \cdots & q_{N-1} & q_{N}
\end{array}\right]^{T}
$$

In this paper the heat flux is approximated by piecewise constants. In the linear IHCP the true temperature vector $\mathbf{T}$ is related to the heat flux vector $\mathbf{q}$ by the relation

$$
\mathbf{T}=\mathbf{X q}
$$

where $\mathbf{X}$ is a sensitivity matrix; it is a square matrix with $N$ by $N$ elements and is given by

$$
\mathbf{X}=\left[\begin{array}{cccccc}
X_{1} & 0 & 0 & \cdots & 0 & 0 \\
X_{2} & X_{1} & 0 & \cdots & 0 & 0 \\
X_{3} & X_{2} & X_{1} & \cdots & 0 & 0 \\
\vdots & \vdots & \vdots & \ddots & 0 & 0 \\
X_{N-1} & X_{N-2} & X_{N-3} & \cdots & X_{1} & 0 \\
X_{N} & X_{N-1} & X_{N-2} & \cdots & X_{2} & X_{1}
\end{array}\right]
$$

Notice that this square matrix has the same elements in each column but each successive column is shifted down one. The numerical values for the first column of Eq. (3a) can be found by using a heat flux of unity value. For the approximation of piecewise constant values for the heat flux history, the elements of the sensitivity matrix are given by

$$
X_{1}=\phi_{1}, X_{2}=\phi_{2}-\phi_{1}, \cdots, X_{N}=\phi_{N}-\phi_{N-1}
$$

where $\phi_{n}$ is the dimensionless temperature at location $\tilde{x}$ for a unit surface heat flux at times $t_{1}=\Delta t, t_{2}=2 \Delta t, \cdots, t_{N}=N \Delta t$ or in dimensionless form as, $\tilde{t}_{1}=\alpha \Delta t / L^{2}, \cdots, \tilde{t}_{N}=\alpha N \Delta t / L^{2}$. 
The structure of $\mathbf{X}$ with the zeros in the upper right is an indication of causality which implies that the computed temperature at a time $t_{M}$ is a function only of $q_{M}$ and previous components. More specifically, the computed temperature at time $t_{M}$ is not a function of the future components $q_{M+1}, \cdots, q_{N}$. Although this statement is true, in all IHCP solution methods at least some future information is utilized to obtain a given heat flux component.

\section{Sensitivity coefficients}

The components of the sensitivity matrix, $\mathbf{X}$, given by Eq. (3a) in addition to being equal to the differences of dimensionless temperature for a constant heat flux are the partial derivatives of the temperature with respect to the heat flux. For example, $X_{1}$ is equal to

$$
X_{1}=\frac{\partial \tilde{T}_{1}}{\partial q_{1}}=\frac{\partial \tilde{T}_{2}}{\partial q_{2}}=\cdots=\frac{\partial \tilde{T}_{N}}{\partial q_{N}}, \tilde{T}_{i}=\frac{T\left(x=L / 2, t_{i}\right)}{\frac{q_{0} L}{k}}
$$

and $X_{j}$ is

$$
X_{j}=\frac{\partial \tilde{T}_{j}}{\partial q_{1}}=\frac{\partial \tilde{T}_{j+1}}{\partial q_{2}}=\cdots=\frac{\partial \tilde{T}_{N}}{\partial q_{N-j+1}}
$$

The dimensionless temperatures for the X21B10T0 and X22B10T0 cases are, [20, 21]

$$
\begin{aligned}
& \tilde{T}_{\text {Х21В10T0 }}(\tilde{x}, \tilde{t})=1-\tilde{x}-2 \sum_{m=1}^{m_{\max }} \frac{\cos \left(\beta_{m} \tilde{x}\right)}{\beta_{m}^{2}} e^{-\beta_{m}^{2} \tilde{t}}, \beta_{m}=\left(m-\frac{1}{2}\right) \pi, m=1,2, \cdots \\
& \tilde{T}_{\text {Х22B10T0 }}(\tilde{x}, \tilde{t})=\tilde{t}+\frac{1}{3}-\tilde{x}+\frac{\tilde{x}^{2}}{2}-2 \sum_{m=1}^{m_{\max }} \frac{\cos \left(\beta_{m} \tilde{x}\right)}{\beta_{m}^{2}} e^{-\beta_{m}^{2} \tilde{t}}, \beta_{m}=m \pi, m=1,2, \cdots
\end{aligned}
$$

These solutions are the responses in the Cartesian domain for a unit step in surface heat flux at $x=0$ while the boundary at $x=L$ is maintained at zero temperature (Eq. (5a)) or zero heat flux (Eq. (5b)). The X21 case approaches a steady state to within about $1 \%$ by the dimensionless

time of $\tilde{t}_{X 21, s s} \approx 2$ and the X22 approaches a quasi-steady state about $\tilde{t}_{X 22, \mathrm{ss}} \approx 0.5$, a factor of 4 smaller. The maximum temperatures for the approach of steady state in Equation (5a) and the approach to quasi-steady state in Eq. (5b) are both about unity.

Sensitivity coefficients are found from Eqs. (5a) and (5b) using Eq. (3b). These sensitivity coefficients can be interpreted physically as the response in the Cartesian domain to a unit impulse in heat flux. That is, a constant heat flux of magnitude unity which persists from 
time $t$ to time $t+\Delta t$. Some of the sensitivity coefficients are displayed in Fig. 1 which shows curves for the dimensionless time steps of $\Delta \tilde{t}=\alpha \Delta t / L^{2}=0.02$ and 0.05 . The first column of Eq. (3a) is for the unit heat flux impulse for the interval $0<\tilde{t}<\Delta \tilde{t}$ and the second column of Eq. (3a) is for the unit impulse heat flux for the interval $\Delta \tilde{t}<\tilde{t}<2 \Delta \tilde{t}$. The upper set of four curves in Fig. 1 is for columns 1 and 2 for X21 and X22. Column 2 has the same shape as column 1 but is displaced one step in time compared to column 1. The curves for cases X21 and X22 have the same time-variation until $\Delta \tilde{t}=0.1$ and then begin quite different shapes. The X21 coefficients rise to a maximum of about 0.047 and then drops toward zero while the X22 coefficients go to the constant value of about 0.05 . These values can be compared with the dimensionless temperature rise which in this paper is about unity.

Equation (3b) shows that the sensitivities have the same units as $\phi_{n}$ and thus can be compared with the maximum temperature rise. One of the principles of parameter estimation is that the sensitivity coefficients should be large and uncorrelated. The IHCP becomes ill-posed when the sensitivity coefficients are small or correlated or both. In fact, the coefficients become both small and correlated as the time steps are made small. Examination of the columns of Eq. (3a) can reveal the conditions of being small or uncorrelated; see Fig. 1. The sensitivity coefficients for dimensionless time steps of $\Delta \tilde{t}=\alpha \Delta t / L^{2}=0.02$ and 0.05 have maximum values of 0.02 and 0.05 , respectively; both of these are small compared to a temperature of unity with the former much more so. The column 1 and 2 coefficients for X22 and $\Delta \tilde{t}=0.05$ become perfectly correlated after about time $\tilde{t} \approx 0.3$. Using solely measurements after that time, it is not possible to independently estimate $q_{1}$ and $q_{2}$. By using smaller times for X22 and $\Delta \tilde{t}=0.05$ it is possible (with regularization) to estimate these components; however, it is difficult and regularization must be utilized. For the dimensionless time step of 0.02, increased delay and damping effects and smaller final magnitude effects are even more marked; see the lower curves in Fig. 1.

Further insight can be obtained from the sensitivity coefficients. First, notice that the value of the coefficients are zero before the initiation of heating. This is an indication of causality and implies that all the temperature measurements earlier than $\tilde{t}_{i-1}$ for a heating component $q_{i}$ in the interval $\tilde{t}_{i}-\tilde{t}_{i-1}$ are independent of $q_{i}$ and subsequent heat flux components. However, the point is confusing because estimation of the heat flux $q_{i}$ usually requires 
measurements after time $\tilde{t}_{i}$, which implies that future information is required. The necessary future time duration is related to the times to reach steady state in the X21 cases and to the quasisteady in the X22 cases. Hence future and past information is needed but not all past and future values are necessarily needed; the concept of "local” regularization is important here [22, 23].

\section{General results for filter formulations}

Some insight for various methods can be obtained by direct application of the $\hat{\mathbf{q}}=\mathbf{F Y}$ relation where $\hat{\mathbf{q}}$ is the estimated heat flux vector and $\mathbf{F}$ is the filter matrix, which is described more fully below. The filter coefficient formulation is not a new method, but rather a new lens through which many existing methods can be examined. Furthermore, for some methods, such as Tikhonov regularization, the filter formulation interpretation allows a method that is actually a whole-domain method to be deployed in a sequential fashion. This facilitates application for near real-time computation of heat fluxes from a continuing stream of temperature data, such as might be used to monitor a continuous, on-line process.

For a single heat flux history and a single interior temperature sensor, the heat flux at a middle time denoted $t_{M}$ is given by

$$
\hat{\mathbf{q}}\left(t_{M}\right)=\hat{q}_{M}=\mathbf{F}\left(t_{M}\right) \mathbf{Y}=\mathbf{f}_{M} \mathbf{Y}
$$

where

$$
\begin{aligned}
& \mathbf{f}_{M}=\left[\begin{array}{lllllll}
f_{M N} & f_{M, N-1} & f_{M, N-2} & \cdots & f_{M 2} & f_{M 1}
\end{array}\right] \\
& \mathbf{Y}^{T}=\left[\begin{array}{llllll}
Y_{1} & Y_{2} & Y_{2} & \cdots & Y_{N-1} & Y_{N}
\end{array}\right]
\end{aligned}
$$

Then the heat flux component in Eq. (6a) is given by

$$
\begin{aligned}
\hat{q}_{M} & =\sum_{i=1}^{N} f_{M, N-i+1} Y_{i}=f_{M, N} Y_{1}+f_{M, N-1} Y_{2}+\cdots+f_{M, N-M+1} Y_{M} \\
& +\cdots+f_{M, 2} Y_{N-1}+f_{M, 1} Y_{N}
\end{aligned}
$$

For some regularization methods, the first few and last few filter coefficients are nearly equal to zero. For those cases, the filter equation can be written as

$$
\begin{aligned}
\hat{q}_{M} & =\sum_{n=-m_{f}}^{m_{p}} f_{n} Y_{M-n}=f_{-m_{f}} Y_{M+m_{f}}+f_{-m_{f}+1} Y_{M+m_{f}-1}+\cdots+f_{0} Y_{M} \\
& +\cdots+f_{m_{p}-1} Y_{M-m_{p}+1}+f_{m_{p}} Y_{M-m_{p}}
\end{aligned}
$$

In matrix form this equation is 


$$
\hat{q}_{M}=\left[\begin{array}{llllll}
f_{m_{p}} & f_{m_{p}-1} & \cdots & f_{0} & \cdots & f_{-m_{f}}
\end{array}\right]\left[\begin{array}{llllll}
Y_{M-m_{p}} & \cdots & Y_{M} & \cdots & Y_{M+m_{f}}
\end{array}\right]^{T}
$$

Insightful relations are now found based on the concept that Eq. (6d) must apply for all conditions, both steady and transient. Let the temperature $Y_{i}$ be equal to a constant in time and equal to $T_{C}$ which permits Eq. (6d) to be written as

$$
\hat{q}_{M}=T_{C} \sum_{n=-m_{f}}^{m_{p}} f_{n}
$$

Two special cases are now considered. The first is for a homogeneous plate with the unknown heat flux at $x=0$ and perfectly insulated at $x=L$. This problem using heat conduction notation is denoted X22B?0T0; the X22 denotes the boundary condition of the second kind at $x=0$ and $L$. The B? 0 denotes an unknown heat flux at $x=0$ and insulation at $x=L$. The initial temperature is zero. See [20] for more on the numbering system. For this X22B?0T0 problem with the temperature constant over time at any point in the plate, the heat flux must be zero; hence for this X22B?0T0 case, the sum of the filter coefficients in Eq. (7a) should be zero,

$$
\left[\sum_{n=-m_{f}}^{m_{p}} f_{n}\right]_{0<x / L<1, \text { Х22В?0ТT }}=0
$$

For a second case, which has a zero temperature at $x=L$ and denoted X21B?0T0, the location of the temperature sensor is important. Let the temperature be the constant (and non-zero) value of $T_{C}$ at a location $x_{s}$. Then the heat flux is given by

$$
\hat{q}=k \frac{T_{C}-0}{\left(L-X_{s}\right)}=\frac{k}{\left(L-X_{s}\right)} T_{C} .
$$

Comparing Eqs. (7a) and (8a), the summation of the filter coefficients for the X21B?0T0 problem must be

$$
\left[\sum_{n=-m_{f}}^{m_{p}} f_{n}\right]_{0<x_{s} / L<1, \text { Х21B?0T0 }}=\frac{k}{\left(L-x_{s}\right)} .
$$

For the special case of a sensor at $x_{s}=L / 2$, the summation of the filter coefficients is

$$
\left[\sum_{n=-m_{f}}^{m_{p}} \tilde{f}_{n}\right]_{X_{s} / L=1 / 2, \text { Х Х1В?०Т } 0}=\frac{L}{k}\left[\sum_{n=-m_{f}}^{m_{p}} f_{n}\right]_{x_{s} / L=1 / 2, \text { Х21В?०Т0 }}=2 .
$$


The results given by Eqs. (7b) and (8b,c) can be employed in the evaluation and intrinsic verification of various IHCP methods.

\section{Optimal comparison criteria}

One measure for comparison of various IHCP methods is the mean square error, MSE, (see Eq. (36) of [16])

$$
E\left(R_{q}\right)=E\left[(\hat{\mathbf{q}}-\mathbf{q})^{T}(\hat{\mathbf{q}}-\mathbf{q})\right]
$$

where $\mathbf{q}$ is the vector of the true heat flux components and $\hat{\mathbf{q}}$ is a vector of the estimated heat flux components. The operator $E$ is for determining the expected value, or statistical mean. An objective in comparing each IHCP method is to minimize this MSE with respect to its regularizing parameter. Since the estimated heat flux can be found using different estimation procedures, the minimum for a given case can give insight into the relative strengths of each method.

With the standard statistical assumptions [1, pages 10,11] of additive, zero mean, constant variance and uncorrelated random errors, $E\left(R_{q}\right)$ becomes the sum of bias and random components [16, Eq. (38)],

$$
E\left(R_{q}\right)=E_{q, \text { bias }}+E_{q, \text { rand }}
$$

Again utilizing the standard statistical assumption these expressions yield

$$
\begin{aligned}
E_{q, \text { bias }} & =[(\mathbf{F X}-\mathbf{I}) \mathbf{q}]^{T}[(\mathbf{F X}-\mathbf{I}) \mathbf{q}] \\
& =\mathbf{q}^{T}(\mathbf{F X}-\mathbf{I})^{T}(\mathbf{F X}-\mathbf{I}) \mathbf{q}=\mathbf{q}^{T}(\mathbf{P}-\mathbf{I})^{T}(\mathbf{P}-\mathbf{I}) \mathbf{q}, \quad \mathbf{P}=\mathbf{F X} \\
E_{q, \text { rand }} & =\sigma_{Y}^{2} \operatorname{tr}\left(\mathbf{F}^{T} \mathbf{F}\right)
\end{aligned}
$$

Equation (11) shows that the bias component, $E_{q, b i a s}$, depends upon the errorless heat flux

components, $\mathbf{q}$, and the $\mathbf{P}$ matrix. The random component, $E_{q, r a n d}$, is proportional to the variance of the temperature errors.

For the function specification and Tikhonov IHCP methods, the filter matrix $\mathbf{F}$ has nearly identical terms along the diagonals, deviating only near the beginning and the end of the diagonals. Hence these values along a row can be called the filter coefficients and then it is possible to deal with such a row, rather than the complete matrix. 
In some IHCP methods, the $\mathbf{P}=\mathbf{F X}$ matrix is symmetric (or nearly so) about the main diagonal and also the sum of the elements on a given row is very close to unity. When these two conditions of symmetry and summing to unity are valid, the heat flux bias given by Eq. (11) is nearly zero for both constant and linearly-in-time varying heat fluxes q. These conclusions can be reached by considering Eq. (11) as follows. Consider the case of a constant-in-time heat flux, then all entries of $\mathbf{q}$ are the same. If each row of $\mathbf{P}$ sums to unity, then the product $\mathbf{P q}$ will be equal to Iq, and $E_{q, b i a s}$ will be zero. Now consider a heat flux which is linearly increasing in time. The important feature is that, for a given row of $\mathbf{P}$, values of $\mathbf{q}$ for subsequent times increase at the same rate as values of $\mathbf{q}$ decrease moving backward in time. If the entries for a row of $\mathbf{P}$ are symmetric, then in the product $\mathbf{P q}$, lesser contributions from past times are exactly compensated by greater contributions from future times. Furthermore, when the row of $\mathbf{P}$ sums to unity, the resulting product $\mathbf{P q}$ will be exactly equal to the value of $q_{\mathrm{M}}$ for that row, and this is exactly equal to the value resulting from the multiplication of Iq. Thus, for the linear-in-time heat flux, Eq. (11) indicates that $E_{q, \text { bias }}$ will be zero. Because the heat flux bias given by Eq. (11) is nearly zero for both constant and linear-in-time varying heat fluxes, it is important to investigate cases when the heat flux deviates substantially from being constant or linearly varying with time.

\section{Function specification (FS)}

The function specification method, FS [1], the heat flux is (temporarily) held constant for $r$ future steps; the result the algebraic FS equation, [1, p.127], at time $t_{M}$

$$
\hat{q}_{F S, M}=\frac{\sum_{i=1}^{r}\left(Y_{M+i-1}-\left.\hat{T}_{M+i-1}\right|_{q_{M}=q_{M+1}=\cdots q_{M+r-1}=0}\right) \phi_{i}}{\sum_{i=1}^{r} \phi_{i}^{2}}, \quad \hat{T}_{M+i-1}=\sum_{n=1}^{M+i-1} q_{n} X_{M+i-n}
$$

The first column of the filter coefficients of Eq. (3a) can be obtained by setting all the $Y$ components equal to zero except the $r^{\text {th }}$ value which is set equal to unity. The number of future temperatures, $r$, might be 2,3 , 4, and even 10 or more. The first value obtained from Eq. (13a) is given for $M=1$,

$$
\hat{q}_{1}=\frac{\phi_{r}}{\sum_{i=1}^{r} \phi_{i}^{2}}
$$


This is actually the diagonal component of the filter coefficients denoted $f_{F S,-r+1}$. In general, the matrix notation for the FS filter matrix can be written as

$$
\mathbf{F}_{F S}=\left[\begin{array}{ccccccc}
f_{-r+1} & 0 & \cdots & 0 & \cdots & 0 & 0 \\
f_{-r+2} & f_{-r+1} & \cdots & 0 & \cdots & 0 & 0 \\
\vdots & \vdots & \ddots & \vdots & \cdots & \vdots & \vdots \\
f_{0} & f_{-1} & \cdots & f_{-r+1} & \cdots & 0 & 0 \\
\vdots & \vdots & \cdots & \vdots & \ddots & \vdots & \vdots \\
f_{N-1} & f_{N-2} & \cdots & f_{-1} & \cdots & f_{-r+1} & 0 \\
f_{N} & f_{N-1} & \cdots & f_{0} & \cdots & f_{-r+2} & f_{-r+1}
\end{array}\right]
$$

The "FS" subscripts are omitted for convenience in this equation. This matrix is persymmetric because it is symmetric about the northeast-southwest diagonal. Figure 2 shows some columns of this matrix for the X22 case with $\tilde{x}=0.5, \Delta \tilde{t}=0.05$ and $r=6$; the same curve shape of starting positive and then going negative keeps repeating for successive columns. Table 1 contains numerical values for the first column given in the matrix of Eq. (14) for $r=2$ to 9. As time increases the values go to zero and the sums of the filter coefficients for the X22 case also go to zero (see the last row of Table 1). The columns (and rows) of the $P$ matrix also repeat; the sum of these coefficients is unity and the shapes are nearly symmetric. But better symmetry is displayed by other IHCP methods.

Results for the sensitivity coefficients, filter coefficients and the $p$-vector are shown in Fig. 3. The function specification method for $r=8$ is used for the X22B?0T0 case with $\tilde{x}=x / L=0.5$ and $\Delta \tilde{t}=\alpha \Delta t / L^{2}=0.05$. The sum of the $p$-values is 0.9991 which is very close to the ideal value of unity; this indicates that the function specification method for this case will give accurate results for a heat flux time variation being nearly a constant with time. See Eq. (11).

Two different prescribed heat fluxes are examined using the FS, Tikhonov and SVD methods. The first example is for a step change in the heat flux and the second has quartic time variation $[16,17]$. The results for the step change case for the FS method are depicted in Fig. 4 for which the location of the sensor is at $\tilde{x}=0.5$ and the time steps are $\alpha \Delta t / L^{2}=0.05$. The plate is insulated at $x=L$ so that the X22 case is utilized. Figure 4 shows results for which $r=4$ future times gives the best results for Gaussian additive, constant variance and uncorrelated errors with a standard deviation of $\sigma_{Y}=0.01$ which is about $1 \%$ of the maximum temperature in 
the examples. This is a very challenging case. It is awkward to plot the true heat flux because it is neither 0 nor 1 at the step; instead the value of 0.5 is plotted. The sum of squares of the errors in the heat fluxes is 0.228 It should be noted the results with error depend upon the particular set of random numbers chosen; for consistency each example uses the same set of random numbers (\#651 in Matlab) and also uses about the same number of measurements for a given example. This FS result is not as good as some given by other methods.

The second series of problems is for a quartic heat flux distribution [16, 17]. The true and estimated FS heat flux histories are displayed in Fig. 5. With the fluctuations associated with $\sigma_{Y}=0.1$ it is remarkable that the shape of the quartic heat flux history is determined as accurately as it is. The optimal number of future times (determined by the minimal sum of squares of the heat flux errors) is $r=5$ but $r=6$ (sum $=0.0986$ ) gives almost the same sum of squares (0.1067). In an aside, the Morozov criterion [16] of reducing the standard deviation of temperature errors to the real value gives $r=6$.

\section{Tikhonov regularization}

The $i^{\text {th }}$ order Tikhonov IHCP procedure produces the estimated heat flux $\hat{\mathbf{q}}$ as

$$
\hat{\mathbf{q}}_{T}=\left(\mathbf{X}^{T} \mathbf{X}+\alpha_{T, i} \mathbf{H}_{i}^{T} \mathbf{H}_{i}\right)^{-1} \mathbf{X}^{T} \mathbf{Y}=\mathbf{F}_{T, i} \mathbf{Y}, \quad \mathbf{F}_{T, i}=\left(\mathbf{X}^{T} \mathbf{X}+\alpha_{T, i} \mathbf{H}_{i}^{T} \mathbf{H}_{i}\right)^{-1} \mathbf{X}^{T}
$$

where for $0^{\text {th }}, 1^{\text {st }}$, and $2^{\text {nd }}$ order regularization [1],

$$
\mathbf{H}_{0}=\mathbf{I}, \quad \mathbf{H}_{1}=\left[\begin{array}{cccccc}
-1 & 1 & \cdots & 0 & 0 & 0 \\
0 & -1 & \cdots & 0 & 0 & 0 \\
\vdots & \vdots & \ddots & \vdots & \vdots & \vdots \\
0 & 0 & \cdots & -1 & 1 & 0 \\
0 & 0 & \cdots & 0 & -1 & 1 \\
0 & 0 & \cdots & 0 & 0 & 0
\end{array}\right], \quad \mathbf{H}_{2}=\left[\begin{array}{cccccc}
1 & -2 & 1 & 0 & \cdots & 0 \\
0 & 1 & -2 & 1 & \ddots & 0 \\
\vdots & \vdots & \ddots & \ddots & \ddots & 0 \\
0 & 0 & \cdots & 1 & -2 & 1 \\
0 & 0 & \cdots & 0 & 0 & 0 \\
0 & 0 & \cdots & 0 & 0 & 0
\end{array}\right]
$$

where $\alpha_{T, i}$ is the $i^{\text {th }}$ order Tikhonov regularization coefficient. The $\mathbf{F}_{\mathrm{T}, i}$ symbol is for Tikhonov regularization filter, hence the T subscript, and $i$ is for the regularization order. Figure 6 shows a typical zeroth order Tikhonov filter, $f$, and $p$ vectors for the $\tilde{x}=0.5$ and $\alpha \Delta t / L^{2}=0.05$ for the $\mathrm{X} 22$ case with $\alpha_{T, 0}=0.02$. These curves are almost identical except in the beginning and end of the $\mathbf{F}_{\mathrm{T}, i}$ matrix. The sum of the $f$-vector components is 0.0043 which is a small number, as it should be, and the sum of the $p$-components is 0.9993 which is very close to unity, also as it 
should be. Furthermore the $p$-curve is quite symmetric, much more so than the function specification results.

Tikhonov regularization can be $0^{\text {th }}, 1^{\text {st }}, 2^{\text {nd }}$ or other order or combination of these. Figure 7 shows filter curves for $0^{\text {th }}, 1^{\text {st }}$ and $2^{\text {nd }}$ order regularization for the same location and time steps for the X22 case. Each case is optimal for $\sigma_{Y}=0.01$. The respective $\alpha_{T, i}$ values are $0.011,0.124$ and 1.6 for $i=0,1$ and 2. The curves in Fig. 7 have a lot of similarities. For example, the sums of the respective filter coefficients are $-0.0002,-0.0001$ and 0.0069 which are close to zero. For each of these cases the $p$-values (not shown, but Fig. 6 shows $p$-values for $i=0$ ) sum to nearly unity (0.99997, 0.99996 and 1.00055$)$ and appear to be symmetric.

Estimated heat fluxes are shown in Fig. 8 for the step change of heat flux example with $0^{\text {th }}$ order Tikhonov regularization and optimal value of $\alpha_{T, 0}=0.0014$; the sum of squares of the $q-$ errors is 0.9791 . The greatest errors are at the end of the time period; this is because the sensitivity coefficients are smaller near the end time and also the $0^{\text {th }}$ order regularization biases the estimated heat flux to zero. Graphical results for the same case with $1^{\text {st }}$ order regularization with the optimal $\alpha_{T, 1}=0.0031$ are shown in Fig. 9; now the sum of squares is 0.2431 . To a significant extent, this is substantially caused by the reduced errors near the end; they are mainly reduced by the "flattening" biasing effect of $1^{\text {st }}$ order regularization. See Table 2a which summarizes results; this is the lowest sum of squares and the best results of those obtained for the step heat flux case.

Before proceeding to give further results for the test cases, details for finding the optimal regularizing parameters are described. Figure 10 illustrates the components in the sum given by Eq. (10) to locate an optimal set of regularization parameters. The bias term, given by Eq. (11), is the upward sloping curve labeled $\mathrm{E}_{q, \text { bias }}$. The estimated random error term is computed using Eq. (12); it is labelled $E_{q, \text { rand }}$ and decreases with increasing values of the Tikhonov coefficient. Summing these two components yields a curve, $\mathrm{E}_{\text {q,tot }}$, which has a minimum, about $\alpha_{T, 1}=0.124$ and a sum of squares equal to 0.03055 . For the particular set of chosen random errors, corresponding values are $\alpha_{T, 1}=0.119$ and sum equal to 0.03033 ; see curve labelled "sum e $\mathrm{e}^{2}$." These values of 0.124 and 0.119 are within $4.2 \%$ of each other and the sums of squares are even 
closer, about $0.7 \%$. This indicates that the selection of the Tikhonov coefficient is relatively insensitive.

A well-known method of finding the optimal regularization parameter is the Morozov principle [16] which reduces the estimated standard deviation of the temperature errors, $s_{Y}$, down to the level consistent with the noise in the data. In this case it is the standard deviation value of $\sigma_{Y}=0.01$. That happens in Fig. 10 about $\alpha_{T, 1}=0.32$ for which the total sum of squares is increased to about 0.05 , which is not far from the optimal sum of about 0.031 . The vertical axis in Fig. 10 has different units for the $s_{Y}$ curve than the expected sums curves.

Figure 11 depicts estimated results for the quartic example for the same location, time step and $\sigma_{Y}$ values. It is for $1^{\text {st }}$ order Tikhonov regularization; the optimal value of $\alpha_{T, 1}=0.119$ (about 10 times larger than the $0^{\text {th }}$ order value) gives the smaller sum of $q$-errors of 0.0303 . In the nearly linearly increasing and decreasing regions of the flux values in Fig. 11, the errors appear to be particularly small; it is partly a result of the $p$-vector summing to unity and being symmetric.

\section{Conjugate Gradient Method}

The conjugate gradient method is actually a family of methods grown out of Newton iteration or gradient descent optimization. The technique begins with assumed values for the solution, and updates or corrections are applied at subsequent iterations to improve the solution. To minimize the sum-squared error between the measured data, $\mathbf{Y}$, and the linear model in Eq. (2c) for the temperature, updates are applied according to

$$
\mathbf{q}_{k+1}=\mathbf{q}_{k}+\rho_{k} \mathbf{w}_{k}
$$

where the descent direction $\mathbf{w}_{k}$, and the step size, $\rho_{k}$, vary depending on the particular CG method employed. For the Fletcher-Reeves algorithm [24],

$$
\mathbf{w}_{k}=\left\{\begin{array}{cc}
2 \mathbf{X}^{T}\left(\mathbf{Y}-\mathbf{X} \mathbf{q}_{k}\right) & k=1 \\
2 \mathbf{X}^{T}\left(\mathbf{Y}-\mathbf{X} \mathbf{q}_{k}\right) \frac{\left\|2 \mathbf{X}^{T}\left(\mathbf{Y}-\mathbf{X} \mathbf{q}_{k}\right)\right\|^{2}}{\left\|2 \mathbf{X}^{T}\left(\mathbf{Y}-\mathbf{X} \mathbf{q}_{k-1}\right)\right\|^{2}} & k>1
\end{array}\right.
$$

and 


$$
\rho_{k}=-\frac{\left(\mathbf{Y}-\mathbf{X} \mathbf{q}_{k}\right)^{T} \mathbf{X} \mathbf{w}_{k}}{\left\|\mathbf{X} \mathbf{w}_{k}\right\|^{2}}
$$

An important consideration for the CG methods is the stopping criteria which dictates the number of iterations required for a converged solution. Classically, the Morozov discrepancy principle is used as this guide; however, when access to the exact solution is available, the expected error in the estimated heat flux can be used.

Figure 12 shows the heat flux history obtained using the Fletcher-Reeves CG analysis for the step heat flux input. The results in Fig. 12 are computed using direct application of CG method as suggested by Eq. (16a) and not using a filter matrix. The same parameters are used for this analysis as in Figs. 4, 8, and 9: $\alpha t / L^{2}=0.05$ and sensor located at $x / L=0.5$. The minimum of the expected error in the residual of the heat flux (Eqs. (9-12)) is used to determine the optimal number of iterations, and in this case $n_{\text {opt }}=6$. The resulting sum of errors squared $e_{q}^{2}=1.123$. It is seen in Fig. 12 that the CG method delivers poor performance at the end of the time interval. Without the last estimate, the sum of squared errors is about 1.04 .

Figure 13 shows the heat flux history obtained using the Fletcher-Reeves CG analysis for the quartic heat flux input. The same parameters are used for this analysis as in Figs. 5 and 11: $\alpha t / L^{2}=0.05$ and sensor located at $x / L=0.5$. The minimum of the expected error in the residual of the heat flux (Eqs. (9-12)) is used to determine the optimal number of iterations, and, again, in this case $n_{\text {opt }}=6$. The resulting sum of errors squared $e_{q}^{2}=0.0444$.

Filter coefficients can be constructed for CG method in a manner similar to that used for FS: solve the inverse problem using a vector of data which is all zero except for a " 1 " at the middle time location, $t_{\mathrm{M}}$. By applying CG method for each successive time, the full filter matrix for CGM can be computed. The number of iterations required is determined by minimizing the $E\left(\mathbf{R}_{q}\right)$ for a known heat flux input, as outlined previously (Eqs. (9-12)). This requires completing a full sweep of all the rows of the $\mathbf{F}$ matrix for each iteration and then computing the heat flux vector using $\mathbf{F}$, and computing and checking $E\left(\mathbf{R}_{q}\right)$ to determine the number of iterations required.

Figure 14 shows the optimal filter coefficients computed for CGM using the step heat flux input. The coefficients from the middle, middle minus one, and middle plus one rows are shown. The three curves are very similar, but are seen to have subtle differences. The peak values are different, and the shapes of the oscillations at early time values are different. 
Furthermore, since the $f$ coefficients do not damp out to zero quickly, data from the far past and future are needed to compute the middle value of $q_{\mathrm{M}}$. The sums of the $f$ coefficients for each of these rows $(23,24$, and 24$)$ are $0.1639,0.4660$, and 0.0463 , which are not uniformly close to zero as they should be. The sums of the $p$ coefficients for these individual rows are 0.9075 , 0.9344 , and 1.017, which are very approximately equal to one, as they should be. It is not clear that the CG method results in universal filter coefficients that can be used for continuous "near real-time” application such as described in Reference 19.

\section{Singular value decomposition}

Unlike the methods discussed above, the singular value decomposition method [5, 6] produces oscillatory results for $f$ and $p$ in time and space even in the mid-time range. See Fig. 14 which shows the main diagonal (lower curve) values for the filter matrix $\mathbf{F}$ as a function of index along the diagonal. The conditions are the standard ones ( $x / L=0.5$, etc.) for 41 time steps and 14 eigenvalues. The oscillations are significant in magnitude with the oscillations increasing in magnitude toward the beginning and the end; 14 peaks are observed which might be related to the 14 eigenvalues. The curves seem to be symmetric about the middle index. The upper curve in Fig. 15 is for the next diagonal; it is similar to the diagonal results except it is positive rather than negative.

Figure 16 shows the filter coefficients as a function of dimensionless time for the mid column and the two adjacent ones. It has seven peaks which decrease quite slowly in magnitude away from the central time.

The $p$-values are shown in Fig. 17 and again shows significant oscillations over the total time domain shown; if a larger domain is considered, the oscillations continue but the magnitudes gradually decrease. The sum of the $p$-values is about the correct value of unity but the symmetry is not good as in Fig. 17 for Tikhonov regularization.

The step change in heat flux case using SVD is shown in Fig. 18; the results are optimal for the standard conditions shown at the top of the figure. In particular, for $\mathrm{N}=41,14$ eigenvalues minimizes both the $\mathrm{E}_{q, \text { tot }}$ and sum of $q$-errors with the respective sums of 1.070 and 0.927. The results near the jump are relatively accurate compared to the $1^{\text {st }}$ order Tikhonov curve in Fig 9 but not overall. The error near the end is particularly inaccurate and the beginning is 
slightly off too. The beginning and the end are very difficult to treat because the appropriate information is not available as indicated by the sensitivity coefficients.

With significant lead and post times, the SVD method for the quartic case with the simulated measurements gives the excellent results shown in Fig. 19 which uses 91 measurements and $n_{\text {eig }}=12$. The expected sum of squares of errors is 0.03124 and the sum of squares for the same set of random errors as used before is 0.02285. These estimated heat fluxes shown in Fig. 19 are more accurate near the peak heat flux than the Tikhonov $1^{\text {st }}$ order results in Fig. 11 . However, the SVD errors before and after heating appear to be a little larger than the Tikhonov results. The oscillatory results for the SVD method seem to be an inherent characteristic as shown by the filter coefficients. At this time it is not clear how to use the SVD results in a digital filter.

\section{Summary and conclusions}

Two sources of errors in the IHCP methods are the bias introduced by regularization and the effects of random errors. A key to understanding various competing methods is to examine the effects of these errors which, in turn, introduces the filter vector and the $p$-vector. The filter vector can also be used to obtain near real time heat flux estimates which could have application in manufacturing operations and also reentry of rockets.

Four methods are investigated: function specification, Tikhonov, conjugate gradient, and singular value decomposition. Two basic examples are covered, a step increase in heat flux and a quartic variation. Table 2 gives a summary of these cases by comparing the known input heat flux, $q_{i}$, and the estimated heat flux, $\hat{q}_{i}$, for the same set of random errors in the "measured" temperature. A comparison can be made by utilizing the sum of squares of the errors in the heat flux, $e_{q, i}=\hat{q}_{i}-q_{i}$. See the $3^{\text {rd }}$ column is Table $2 \mathrm{a}$ and $4^{\text {th }}$ column in Table $2 \mathrm{~b}$. A crude way to rank the methods uses the relative sum of squares. The last columns in Tables $2 \mathrm{a}$ and $2 \mathrm{~b}$ give the rankings, with the smallest being the best. Adding the scores for the two test cases gives the overall ranking: $1^{\text {st }}$ order Tikhonov, SVD, FS and $0^{\text {th }}$ order Tikhonov/CG (tied). If instead a ranking is based on the sums of $e_{q}{ }^{2}$ for both test cases for each method, the ranking is $1^{\text {st }}$ order Tikhonov, FS, SVD, $0^{\text {th }}$ order Tikhonov, and CG. In both comparisons, the $1^{\text {st }}$ order Tikhonov method is the best. Other test problems would yield different rankings but for the present purposes the $1^{\text {st }}$ order Tikhonov method is the best of the tested methods. Further reasons for this conclusion are given next. 
The $f$ - and $p$-vectors for FS and Tikhonov have desirable sums and shapes. For the conditions denoted by X22 and $\tilde{x}=1 / 2, \alpha \Delta t / L^{2}=0.05$ and $\sigma_{Y}$, the $f$-vector should have a sum of zero and have a minimum time span. The sensitivity coefficients give an indication of the minimum time span. From Fig. 1, the minimum time span is about $\alpha \Delta t / L^{2}=0.3$ but this is only for the future; if the past is included at least the same time span is needed which gives a total time span of $\alpha \Delta t / L^{2}=0.6$. Figure 3 for the FS method and Figs. 6 and 7 for the Tikhonov method both indicate filter coefficient time durations of about twice that value or $\alpha \Delta t / L^{2}=1.2$. However, the end behavior of the step case for $0^{\text {th }}$ order Tikhonov regularization in Fig. 8 seems to indicate that the anomalous time period at the end is about $\alpha \Delta t / L^{2}=0.3$.

Filter coefficients for the CG and SVD methods have undesirable filter characteristics, and it is not clear that they can be used in a true filter form. Their respective non-zero filter coefficients extend both in the past and in the future for large oscillatory periods, and the shapes of the $f(t)$ curves are different at different times. However, both methods yield good results and are competitive techniques for inverse heat conduction problems.

\section{Acknowledgements}

This work was partially supported under NSF grant CBET 1250625. The authors are grateful for this support.

\section{Nomenclature}

$k$ thermal conductivity, W/m-K

$L \quad$ thickness of 1 -D domain, $m$

e error

$E(\cdot) \quad$ expected value

$f \quad$ filter coefficient

f filter vector (a row of the filter matrix)

F filter matrix

$\mathbf{H}_{i}$ Tikhonov regularization matrix of order $I$, Eq. (15b,c,d) 
I identity matrix

$m_{f} \quad$ number of non-zero filter coefficient in the future

$m_{p} \quad$ number of non-zero filter coefficient in the past

$n_{\text {eig }} \quad$ number of eigenvalues retained in SVD method

$\mathrm{N}$ total number of time steps

$\mathbf{P}$ matrix equal to $\mathbf{F X}$

$q_{i} \quad$ heat flux component at time $t_{i}, \mathrm{~W} / \mathrm{m}^{2}$

q vector of heat flux components, $\mathrm{W} / \mathrm{m}^{2}$

$r \quad$ number of future time steps

$R \quad$ residual

$S_{Y} \quad$ estimated standard deviation of temperature errors, $\mathrm{K}$

$t \quad$ time, $\mathrm{s}$

$T_{i} \quad$ true or computed temperature at time $t_{i}, \mathrm{~K}$

$\mathbf{T} \quad$ vector of true or computed temperatures, $\mathrm{K}$

w descent direction

$x \quad$ Cartesian coordinate, $\mathrm{m}$

$\mathbf{X}$ sensitivity matrix, Eq. (3a)

$Y_{i} \quad$ temperature measurement at time $t_{i}, \mathrm{~K}$

$\mathbf{Y} \quad$ vector of measured temperatures, $\mathrm{K}$

\section{Greek}

$\alpha \quad$ thermal diffusivity, $\mathrm{m}^{2} / \mathrm{s}$

$\alpha_{T} \quad$ Tikhonov regularization parameter

$\beta \quad$ eigenvalue

$\varepsilon_{i} \quad$ additive temperature error at time $t_{i}, \mathrm{~K}$

$\boldsymbol{\varepsilon} \quad$ vector of additive temperature error, $\mathrm{K}$

$\phi \quad$ temperature response due to unity heat flux, $\mathrm{K}$

$\rho \quad$ step size

$\sigma \quad$ standard deviation

\section{Subscripts/Superscripts}




$\begin{array}{ll}\text { C } & \text { a constant value } \\ \text { FS } & \text { function specification } \\ f & \text { future } \\ k & \text { iteration index } \\ \text { M } & \text { a middle value (refers to the current time step) } \\ \text { N } & \text { number of time steps; index of the last row of matrix } \\ p & \text { past } \\ q & \text { heat flux } \\ \text { S } & \text { sensor location } \\ \text { ss } & \text { steady-state } \\ \text { T } & \text { Tikhonov } \\ T & \text { temperature }\end{array}$

\section{Decorations}

$\sim \quad$ dimensionless quantity

$\wedge \quad$ estimated value

\section{References}

[1] J.V. Beck, B. Blackwell, C.R. St Clair Jr., Inverse Heat Conduction: Ill-Posed Problems, John Wiley and Sons, New York, (1985).

[2] J. V. Beck, "Surface Heat Flux Determination Using An Integral Method." Nuclear Engineering And Design, 7.(1968): 170-178.

[3] A.N. Tikhonov, V.Y. Arsenin, Solutions of Ill-posed Problems, V.H. Winston \& Sons, Washington, DC, (1977).

[4] A. N. Tikhonov, “On the regularization of ill-posed problems,” Doklady Akademii Nauk SSSR (1963), 153, 49-52

[5] A. N. Tikhonov, "On the solution of ill-posed problems and the method of regularization," Doklady Akademii Nauk SSSR (1963), 151, 501--504

[6] M.N. Ozisik, H.R.B. Orlande, Inverse Heat Transfer, Taylor \& Francis, New York, (2000). 
[7] Y. Jarny, M. N. Ozisik, J.-P. Bardon, “A general optimization method using adjoint equation for solving multidimensional inverse heat conduction,” International Journal of Heat and Mass Transfer, 34(11):2911-2919 (1991)

[8] O.M. Alifanov, Inverse Heat Transfer Problems, Springer-Verlag, Heidelberg, (1994).

[9] O. M. Alifanov, S. V.Rumyantsev, "Application of iterative regularization for the solution of incorrect inverse problems,” Journal of Engineering Physics and Thermophysics, Vol. 53 Issue: 5 p1335-1342, (1987),

[10] O. M. Alifanov, S. V.Rumyantsev, "One method of solving incorrectly stated problems," Journal of Engineering Physics and Thermophysics, Vol. 34 Issue: 2 p223-226, (1978)

[11] P. C. Hansen, “ The truncated SVD as a method for regularization,” BIT Numerical Mathematics 27 (4), 534-553 (1987)

[12] P. C. Hansen, Rank-deficient and discrete ill-posed problems: numerical aspects of linear inversion, SIAM, (1998)

[13] J.R. Shenefelt, R. Luck, R.P. Taylor, J.T. Berry, Solution to inverse heat conduction problems employing singular value decomposition and model reduction, Int. J Heat Mass Transfer 45 (2002), pp. 67-74.

[14] J.M. Gutierrez, J.A. Martin, A. Corz, A sequential algorithm of inverse heat conduction problems using singular value decomposition, Int. J. Therm. Sci. 44(2005), pp. 235-244.

[15] J.V. Beck, Filter solutions for the nonlinear inverse heat conduction problem, Inverse Prob. Sci. Eng. 16 (2008) 3-20.

[16] K. Woodbury, J.V. Beck, Estimation metrics and optimal regularization in a Tikhonov digital filter for the inverse heat conduction problem, Int. J. Heat Mass Transfer, 62, (2013), pp. 31-39.

[17] K. Woodbury, J.V. Beck, "Heat conduction in a planar slab with power-law and polynomial heat flux input," Int. J. of Thermal Sciences, 30, (2013), 1-12.

[18] K. A. Woodbury, J. V. Beck, H. Najafi, Filter solution of inverse heat conduction problem using measured temperature history as remote boundary condition, Int. J Heat Mass Transfer 72 (2014), pp. 139-147

[19] H. Najafi, K. A. Woodbury, J. V. Beck, N. Keltner, "Real-time heat flux measurement using directional flame thermometer”, Applied Thermal Engineering, 86, pp. 229-237, (2015) 
[20] K. Cole, J.V. Beck, A. Haji-Sheihh, B. Litkouhi, Heat Conduction using Green’s Functions, Second Ed., CRC Press, Boca Raton, (2011).

[21] K.D. Cole, K. Woodbury, J. Beck, F. de Monte, D. Amos, A. Haji-Sheikh, et al., Exact Analytical Conduction Toolbox, 2013, www.exact.unl.edu.

[22] P.K. Lamm, T.L. Scofield, Local regularization methods for the stabilization of linear illposed equations of the Volterra type, Numer. Funct. Anal. Optim. 22 (7 \& 8) (2001), pp. 913940.

[23] P.K. Lamm, Full convergence of sequential local regularization methods for Volterra inverse problems, Inverse Prob. 21 (2005), pp. 785-803.

[24] Jarny, Y., and Orlande, H. R. B., “Adjoint Methods” in Thermal Measurements and Inverse Techniques, edited by Orlande, Fudym, Maillet, and Cotta, CRC Press, 2011 
Table 1. Filter coefficients for the function specification method X22 case, $\alpha \Delta t / L^{2}=0.05, x / L$

\begin{tabular}{|c|c|c|c|c|c|c|c|c|}
\hline & f & 3) & 4) & $f$ & 6) & 7) & 8) & \\
\hline & 3 & & & & & & & 7549 \\
\hline & & 22 & 65 & 1 & 5 & 9 & 57 & .5313 \\
\hline & -5.0900 & -2.9895 & -0.6137 & 0.1294 & 0.3445 & 0.3891 & 0.3767 & 3463 \\
\hline 00 & 0.0683 & -2.2520 & -1.4458 & -0.5002 & -0.0795 & 00971 & 0.1687 & 1935 \\
\hline & 0 . & 9 & 5 & -0.8612 & 10 & 0 & 8 & 00674 \\
\hline 300 & 0.0 & -0.3 & -0.7 & -0. & 36 & -0 & & . \\
\hline 0 & -0.0017 & -0.1417 & -0.4075 & -0.5133 & 220 & -0 & 74 & 26 \\
\hline 100 & -0.0017 & -0.0548 & -02 & -0.3448 & -0 & -0 & 31 & 922 \\
\hline 450 & 2 & -0.0 & -0.1351 & -0.2316 & 95 & -0 & 35 & 00 \\
\hline 500 & 0 & 1 & 8 & $-c$ & 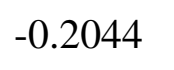 & -( & -0.2316 & -0.2267 \\
\hline טכנ & 00 & -0.0 & -0.0 & -0 . & $-c$ & -0 & 0 & -0.1872 \\
\hline 00 & 0.0000 & -0.0012 & -0.0258 & -0.0701 & -0.1094 & -0 . & 93 & -0 \\
\hline 650 & -0.0000 & -0.0005 & -0.0148 & -0.0471 & -0.0800 & -0.1046 & -0 & -0.1277 \\
\hline 0.700 & -0 . & -0 & -( & $-c$ & -( & $-c$ & -0.0962 & -0.1054 \\
\hline 750 & 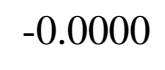 & . & 9 & -0 & 8 & 4 & & 71 \\
\hline 800 & 0.0000 & -0.0000 & -0.0028 & -0.0143 & 13 & -0 . & 20 & 719 \\
\hline 350 & 0.0000 & -0.0000 & -0.0016 & -0.0096 & -0.0229 & -0.1 & -0. & -0.0594 \\
\hline 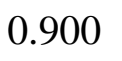 & 00 & -0.0000 & -0.0009 & -0.0064 & -0.0168 & -0 . & & -0 \\
\hline 0.950 & -0.0000 & -0.0000 & -0.0005 & -0.0043 & -0.0123 & -0.0222 & & -0.0405 \\
\hline 1.000 & $-c$ & -0 & - & -( & 0 & 2 & & \\
\hline 1.050 & -0.0000 & -0.0000 & -0.0 & -0.0 & 6 & -0 . & 7 & 276 \\
\hline 1.100 & 0.0000 & -0.0000 & -0.0001 & -0.0013 & -0.0048 & -0.0103 & -0.0166 & -0.0228 \\
\hline 50 & 0.0000 & -0.0000 & -0.0001 & -0.0009 & -0.0035 & -0.0079 & 3 & 0 \\
\hline 1.200 & 0. & -0.0 & -0.0 & -0.00 & -0 & -0 . & 7 & -0 \\
\hline 1.250 & -0.0000 & -0.0000 & -0.0000 & -0.0004 & 19 & -0 & -0 & -0 \\
\hline 1.300 & -0.0000 & -0.0000 & -0.0000 & -0.0003 & 14 & -0 . & -0. & -0.010 \\
\hline 1.000 & -0.0000 & -0.0000 & -0.0000 & -0.0002 & -0.0 & -0.0028 & -0.0055 & -0.00 \\
\hline 1.400 & 0.0000 & -0.0000 & -0.0 & -0 . & -0 & -0 & 4 & -0.007 \\
\hline
\end{tabular}




$\begin{array}{lrrrrrrrr}1.450 & 0.0000 & -0.0000 & -0.0000 & -0.0001 & -0.0005 & -0.0017 & -0.0036 & -0.0060 \\ 1.500 & -0.0000 & -0.0000 & -0.0000 & -0.0001 & -0.0004 & -0.0013 & -0.0029 & -0.0049 \\ 1.550 & -0.0000 & -0.0000 & -0.0000 & -0.0000 & -0.0003 & -0.0010 & -0.0023 & -0.0041 \\ 1.600 & -0.0000 & -0.0000 & -0.0000 & -0.0000 & -0.0002 & -0.0008 & -0.0018 & -0.0034 \\ 1.650 & 0.0000 & -0.0000 & -0.0000 & -0.0000 & -0.0002 & -0.0006 & -0.0015 & -0.0028 \\ 1.700 & 0.0000 & -0.0000 & -0.0000 & -0.0000 & -0.0001 & -0.0005 & -0.0012 & -0.0023 \\ 1.750 & 0.0000 & -0.0000 & -0.0000 & -0.0000 & -0.0001 & -0.0004 & -0.0010 & -0.0019 \\ 1.800 & -0.0000 & -0.0000 & -0.0000 & -0.0000 & -0.0001 & -0.0003 & -0.0008 & -0.0016 \\ 1.850 & -0.0000 & -0.0000 & -0.0000 & -0.0000 & -0.0000 & -0.0002 & -0.0006 & -0.0013 \\ 1.900 & -0.0000 & -0.0000 & -0.0000 & -0.0000 & -0.0000 & -0.0002 & -0.0005 & -0.0011 \\ 1.950 & 0.0000 & -0.0000 & -0.0000 & -0.0000 & -0.0000 & -0.0001 & -0.0004 & -0.0009 \\ 2.000 & 0.0000 & -0.0000 & -0.0000 & -0.0000 & -0.0000 & -0.0001 & -0.0003 & -0.0007 \\ 2.050 & 0.0000 & -0.0000 & -0.0000 & -0.0000 & -0.0000 & -0.0001 & -0.0003 & -0.0006 \\ \text { sum f } & 0.0000 & 0.0000 & 0.0000 & 0.0000 & 0.0000 & 0.0003 & 0.0010 & 0.0029\end{array}$


Table 2. Summary comparison for function specification, Tikhonov and SVD regularization methods. In each case, $x / L=0.5, \alpha \Delta t / L^{2}=0.05$ and $\sigma_{Y}=0.01$.

2a. Summary for various methods for the step change in heat flux example

$\begin{array}{llll}\text { Method } & \text { reg. par. } & \sum e_{q}^{2} & \text { Ranking } \\ \text { Func. Spec. } & r=4 & 0.3136 & 2 \\ \text { Tikhonov, 0th order } & \alpha_{T, 0}=0.0014 & 0.9791 & 4 \\ \text { Tikhonov, 1st order } & \alpha_{T, 1}=0.0031 & 0.2431 & 1 \\ \text { CG } & n_{\text {opt }}=6 & 1.1228 & 5 \\ \text { SVD } & n_{\text {eig }}=14 & 0.9273 & 3\end{array}$

2b. Summary for various methods for quartic heat flux example

Method $\quad$ reg. par. $\quad \mathrm{E}_{q, \text { tot }} \quad \sum e_{q}^{2} \quad$ Ranking

$\begin{array}{lllll}\text { Func. Sp. } & r=5 & & 0.0986 & 5 \\ \text { Tik, order 0 } & \alpha_{T, 0}=0.0115 & 0.0986 & 0.0935 & 4 \\ \text { Tik, order } 1 & \alpha_{T, 1}=0.119 & 0.0305 & 0.0303 & 2 \\ \text { CG } & n_{\text {opt }}=6 & -- & 0.0444 & 3 \\ \text { SVD } & n_{\text {eig }}=12 & 0.0312 & 0.0229 & 1\end{array}$




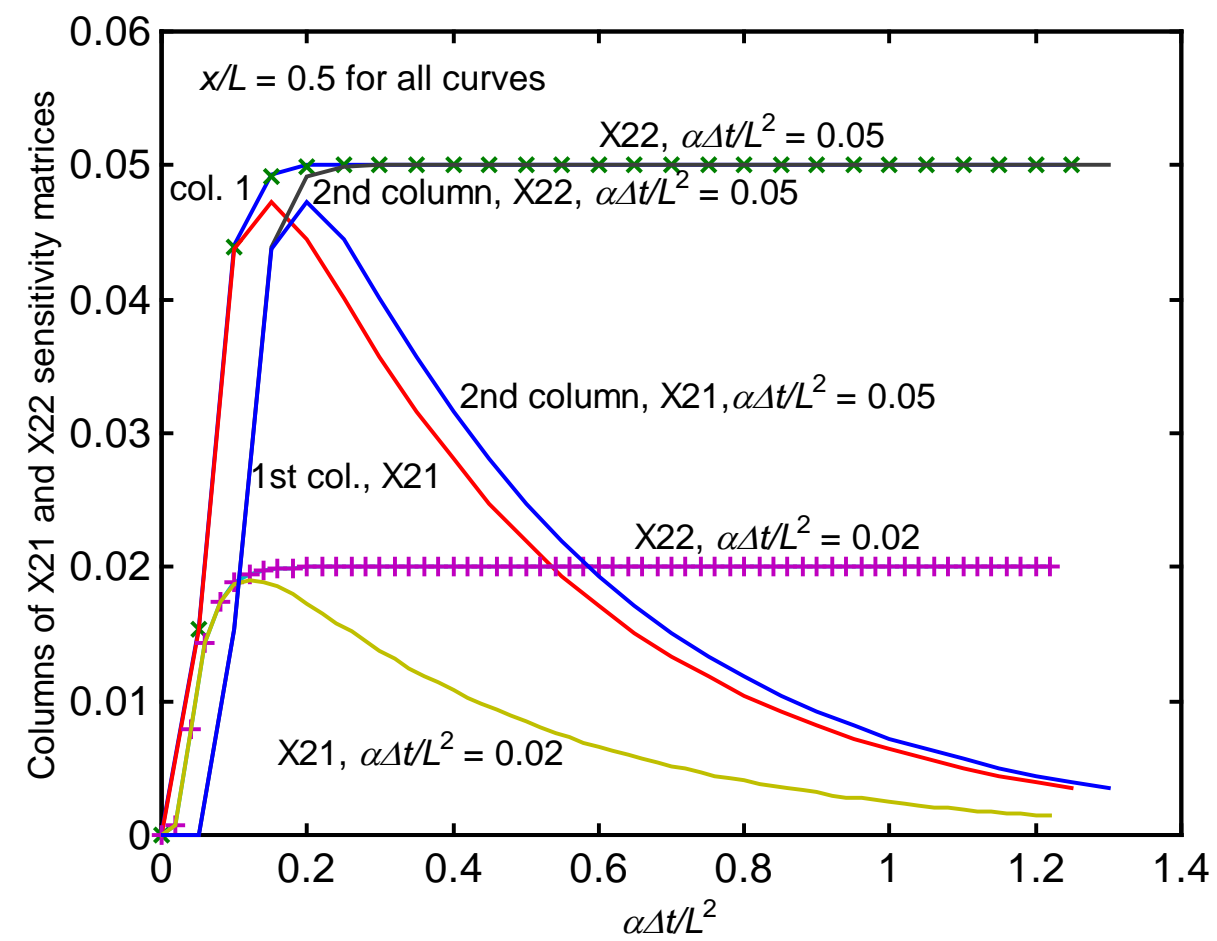

Fig. 1. Columns 1 and 2 of the X matrix X21 and X22 sensitivity coefficients for $x / L=0.5$ with $\alpha \Delta t / L^{2}=0.02$ and 0.05. ihcpstolzX21.m iplot $==1$ 


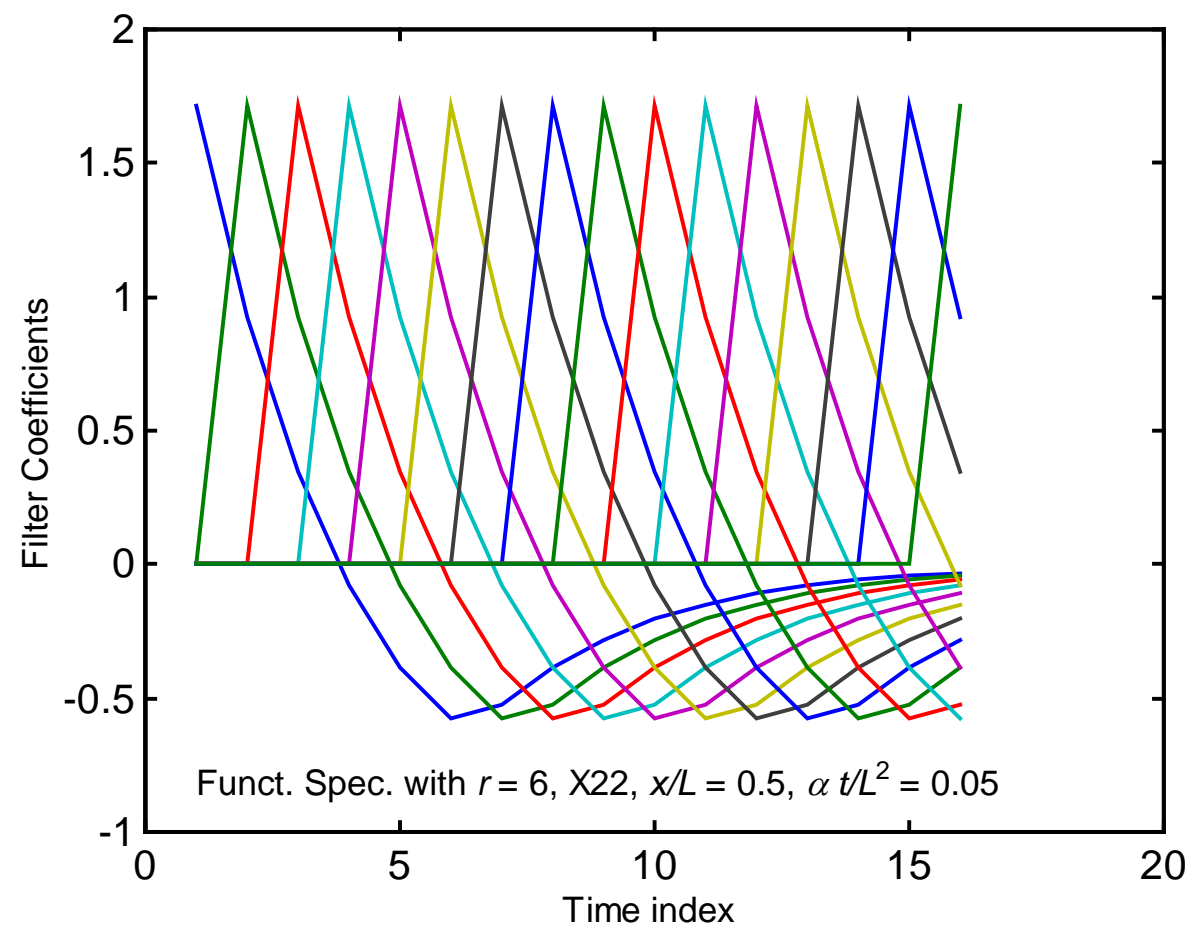

Fig. 2. Filter coefficients for function specification method for X22 case with $r=6, x / L=0.5$ and $\alpha \Delta t / L^{2}=0.05$. funcspecp5compa.m iplot $=1, \operatorname{maxN}=16$ 


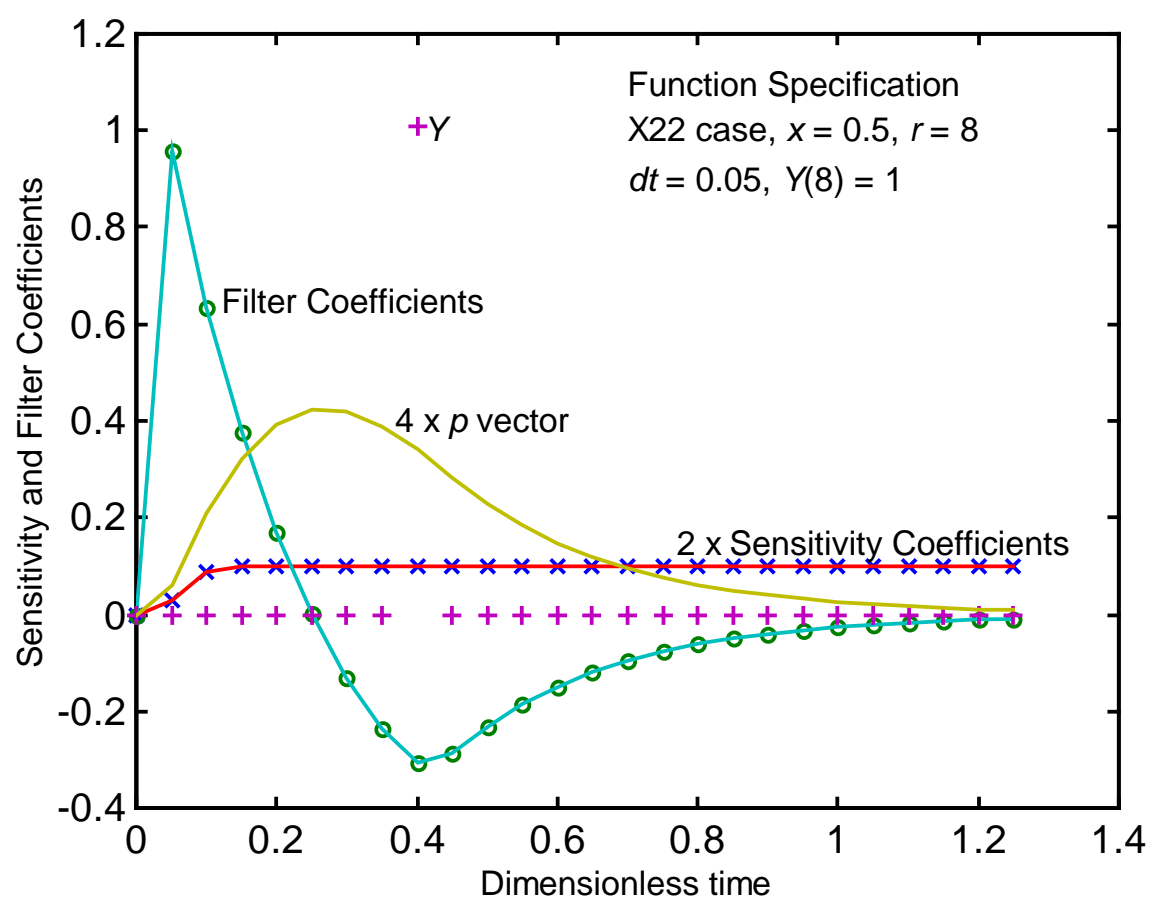

Fig. 3. Sensitivity and filter coefficients and $p$-vector for the function specification method for the X22B?0T0 case with $r=8, \tilde{x}=1 / 2, \Delta \tilde{t}=0.05$ and $\sigma_{Y}$. The "measured" temperatures are all zero except the $r=8^{\text {th }}$ value which is unity; see the + symbols. funcspecp5.m icase $=2$ and $\mathrm{dt}=$ 0.05 


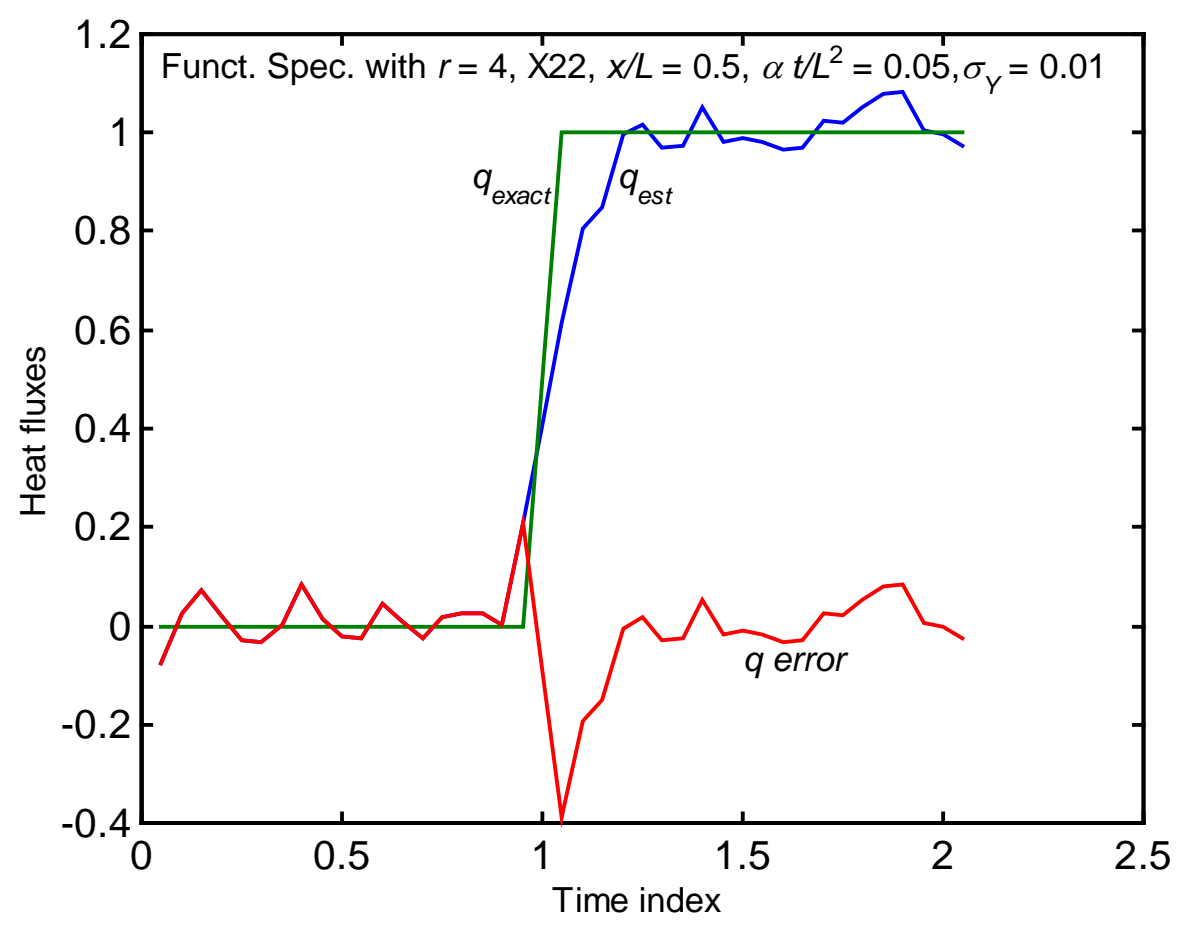

Fig. 4. Estimated heat flux using the function specification method with $r=4$ and $\sigma_{Y}=0.01, x / L=0.5, \alpha \Delta t / L^{2}=0.05$ 


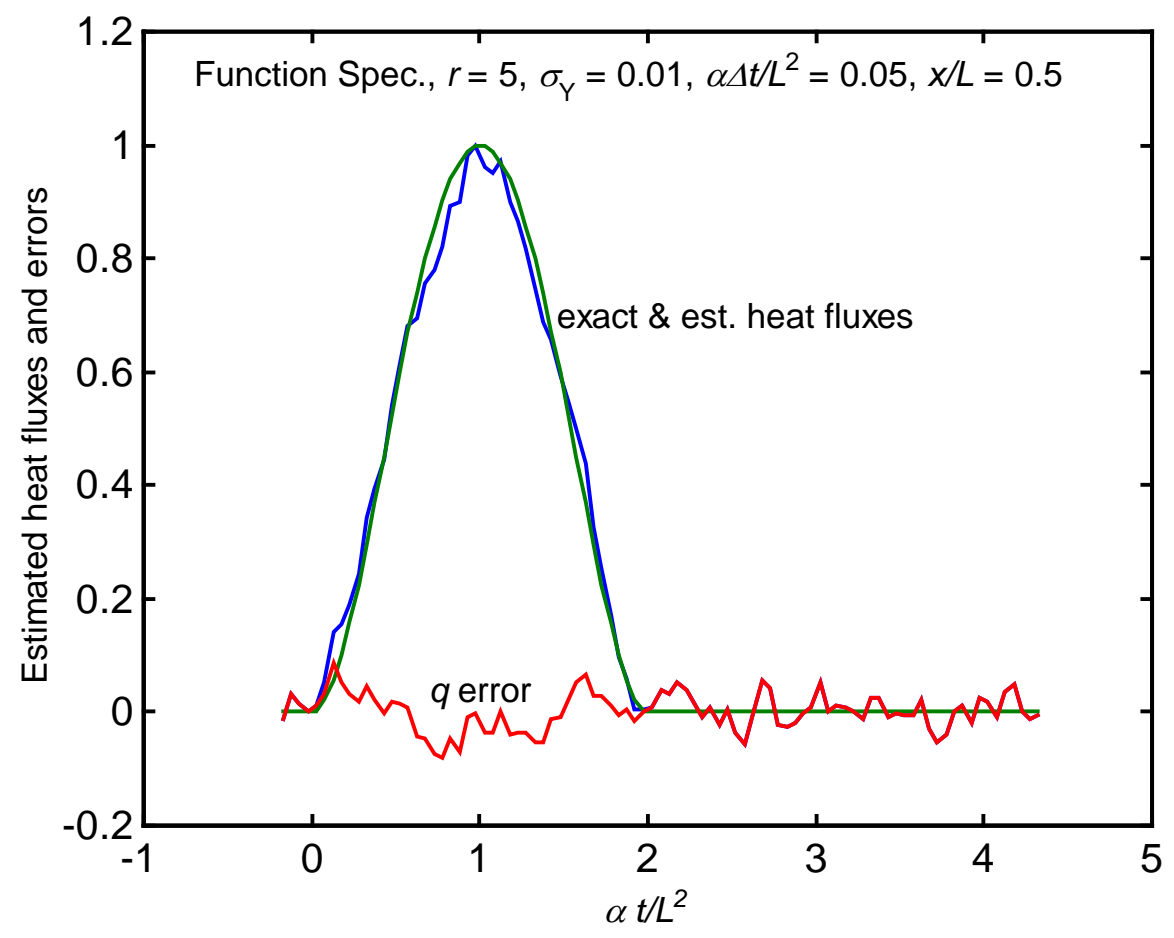

Fig. 5. Function specification with $r=5$ estimated heat flux for quartic heat flux case. $x / L=0.5, \alpha \Delta t / L^{2}=0.05, \sigma_{Y}=0.01$ funcspecquartic.m 


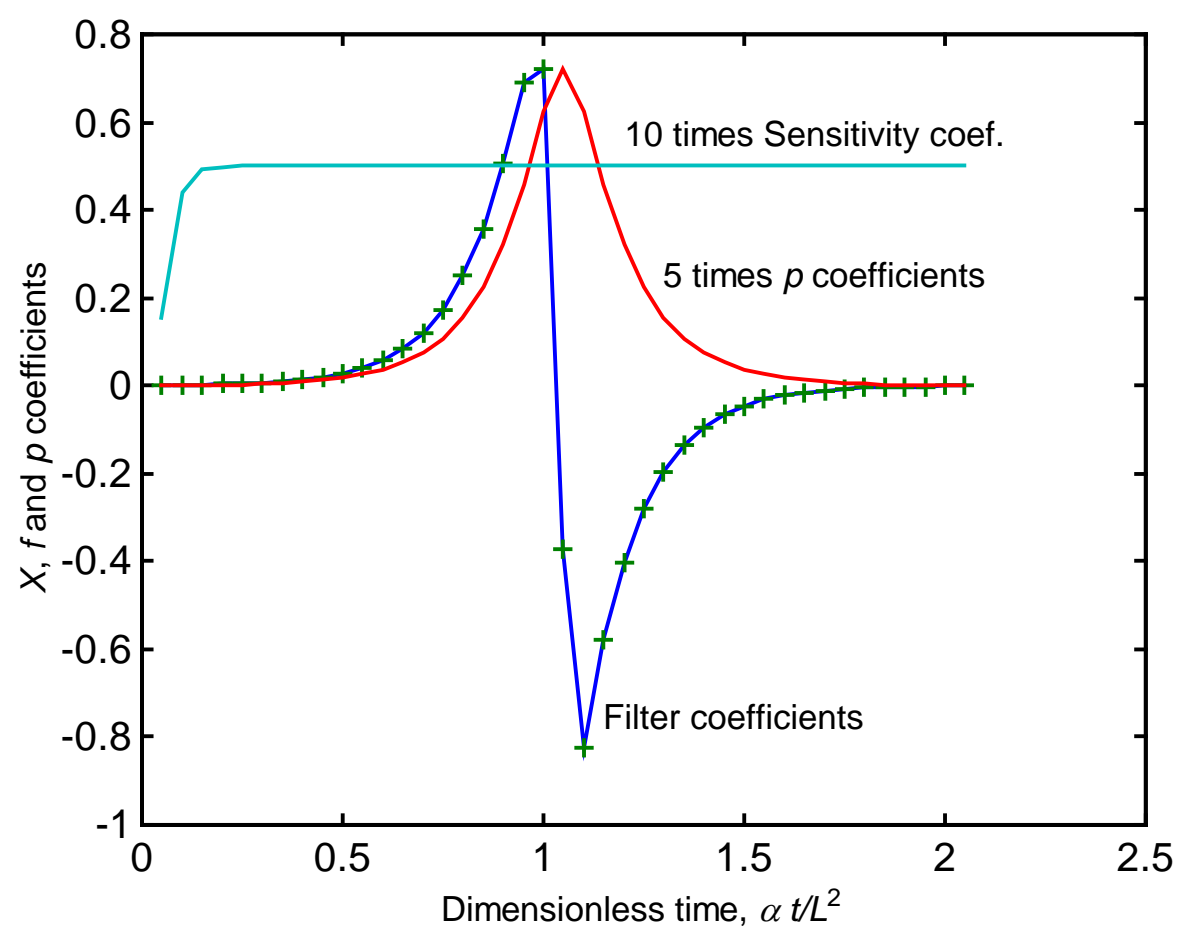

Fig, 6. Zeroth order Tikhonov $f$ and $p$ coefficients for the X22 case $\tilde{x}=0.5, \alpha \Delta t / L^{2}=0.05$ $\alpha_{T, 0}=0.02$ Tikhonov.m iplot $=1$ order $=0$. 


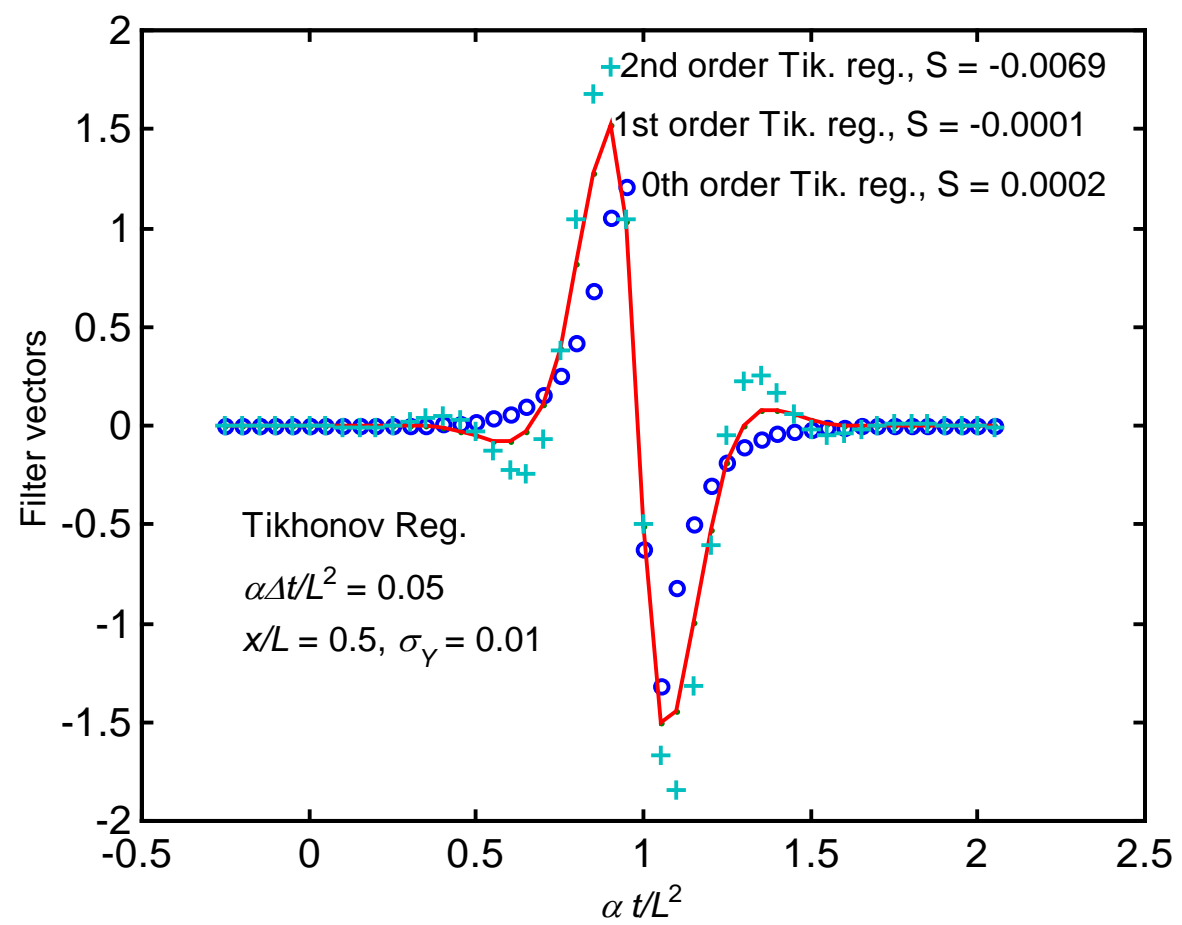

Fig. 7. Zeroth, first and second order Tikhonov filter vectors for optimal cases for $\sigma_{Y}=0.01, \tilde{x}=0.5, \alpha \Delta t / L^{2}=0.05$ and X22 case. The Tikhonov coefficients are 0.011, 0.124 and 1.6, respectively. $S=$ sum filter coefficients. 


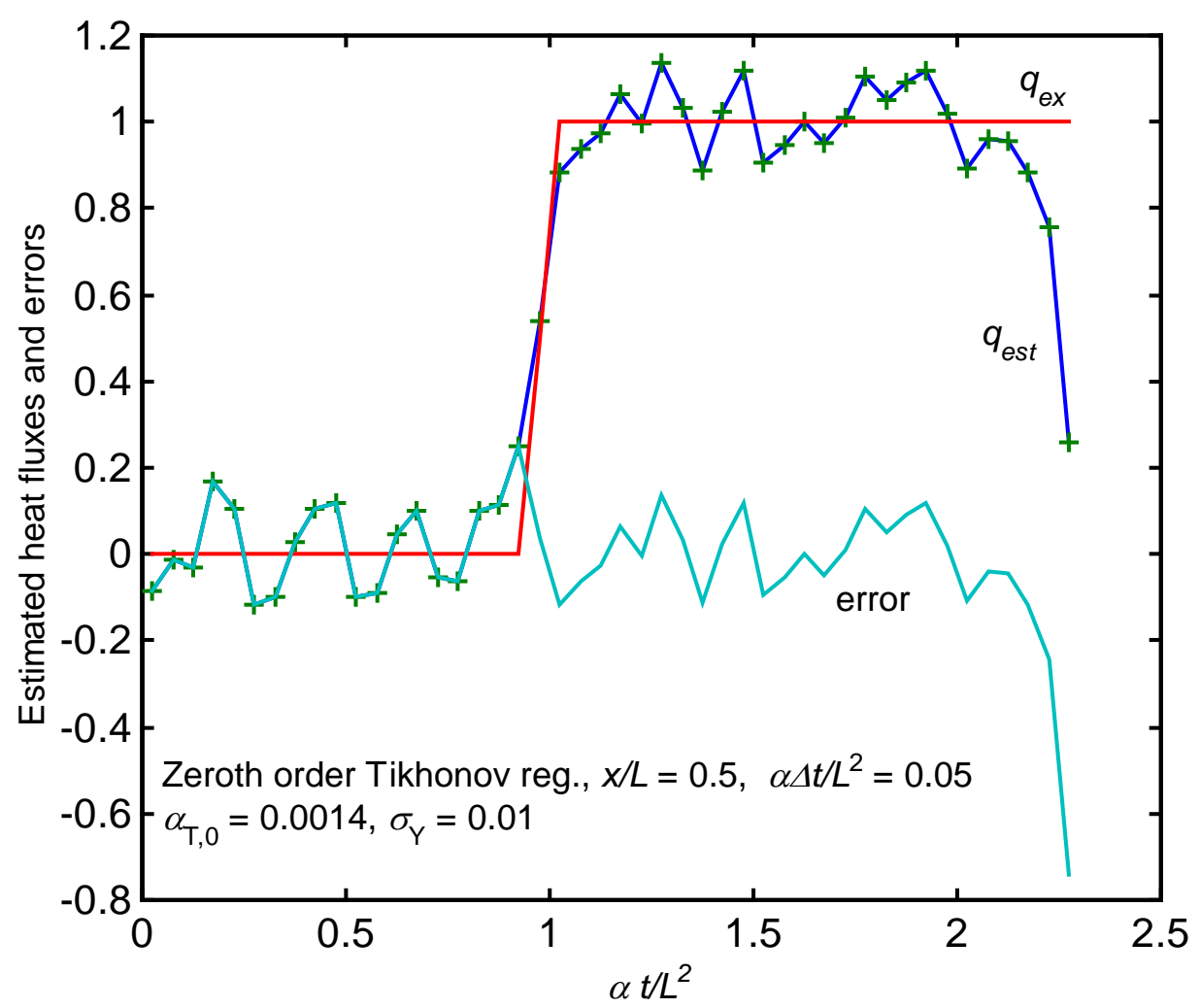

Fig. 8. Heat flux estimates and errors for step heat flux using zeroth order Tikhonov filter coefficients. Tikhonovstep. $\mathrm{m}$ iplot $=2,0^{\text {th }}$ order alphaT $=0.0014$. 


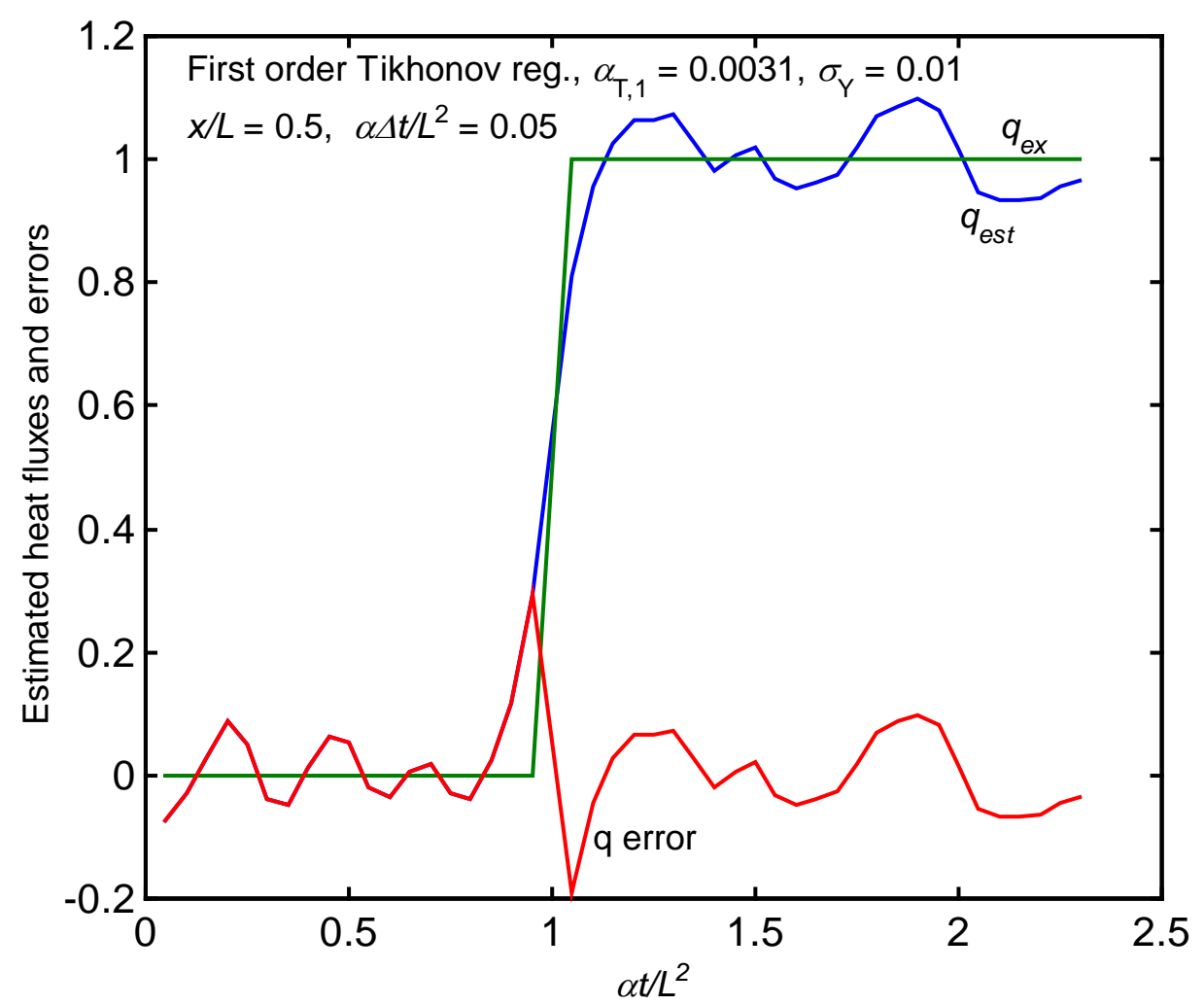

Fig. 9. Heat flux estimates and errors for step heat flux using first order Tikhonov filter coefficients. Tikhonov, $1^{\text {st }}$ order, step change in heat flux, sum of squares of errors $=0.24308$. 


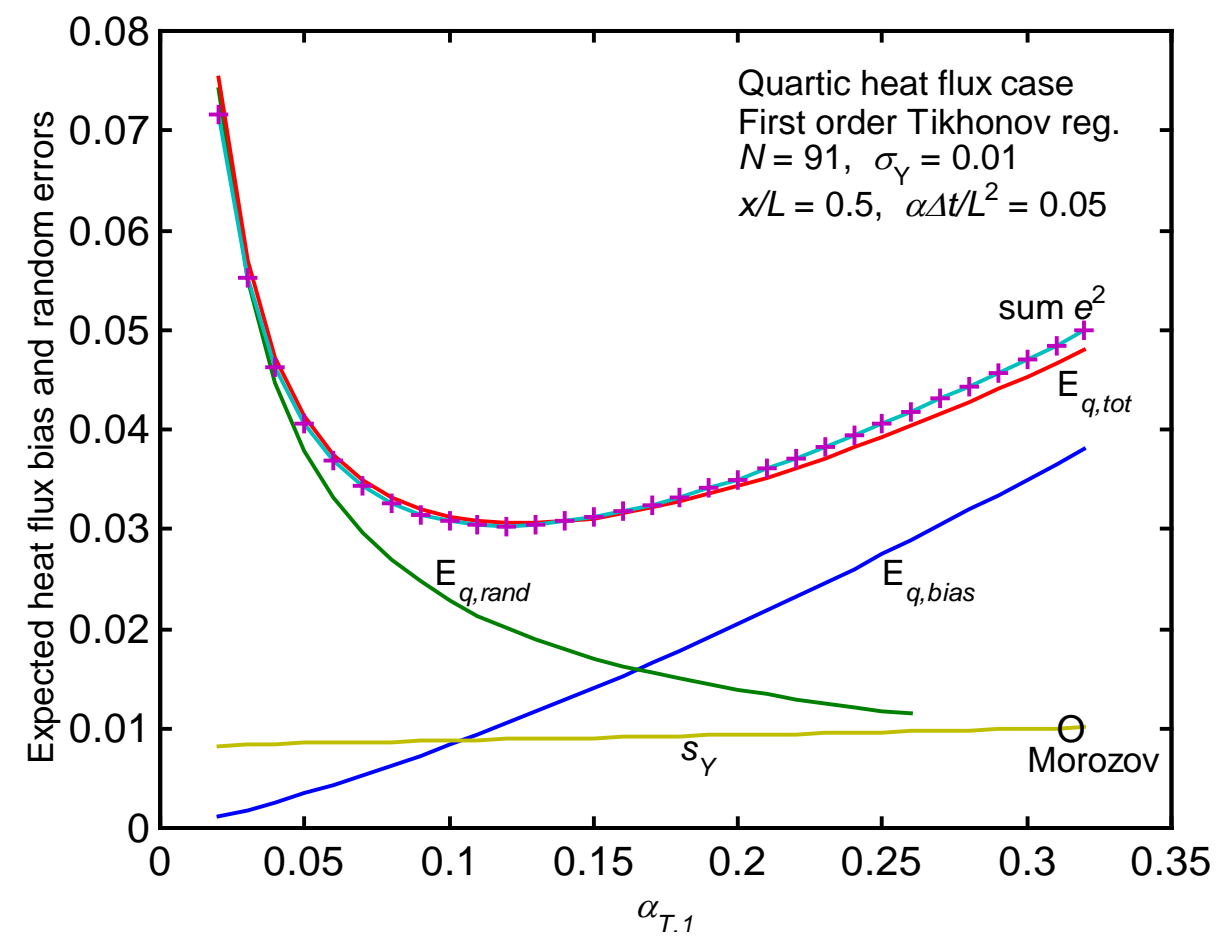

Fig. 10 Components of expected error for determining optimal Tikhonov first order regularization for the quartic heat flux case. 


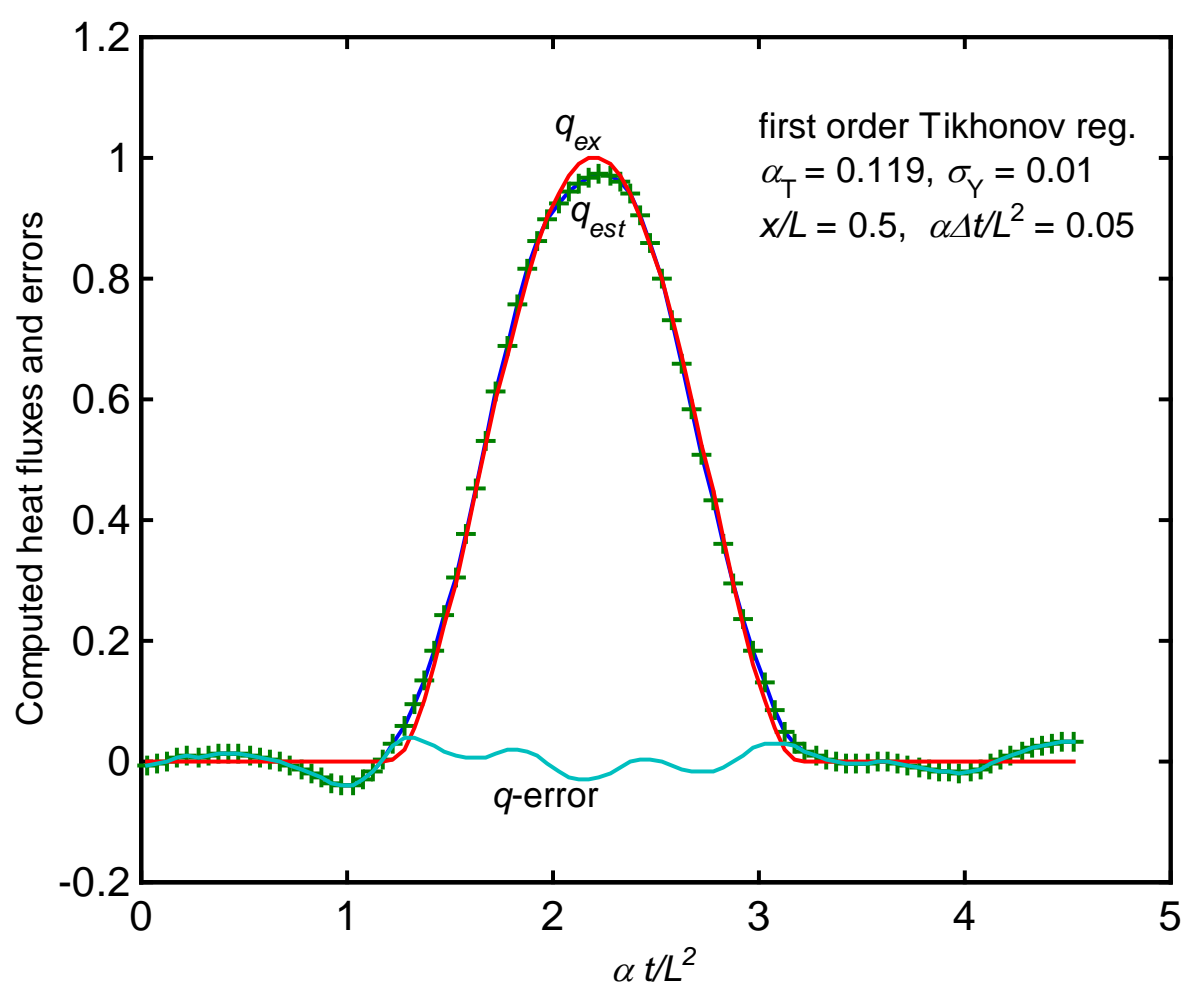

Fig. 11. Heat fluxes and error for the quartic heat flux using first order Tikhonov regularization. 


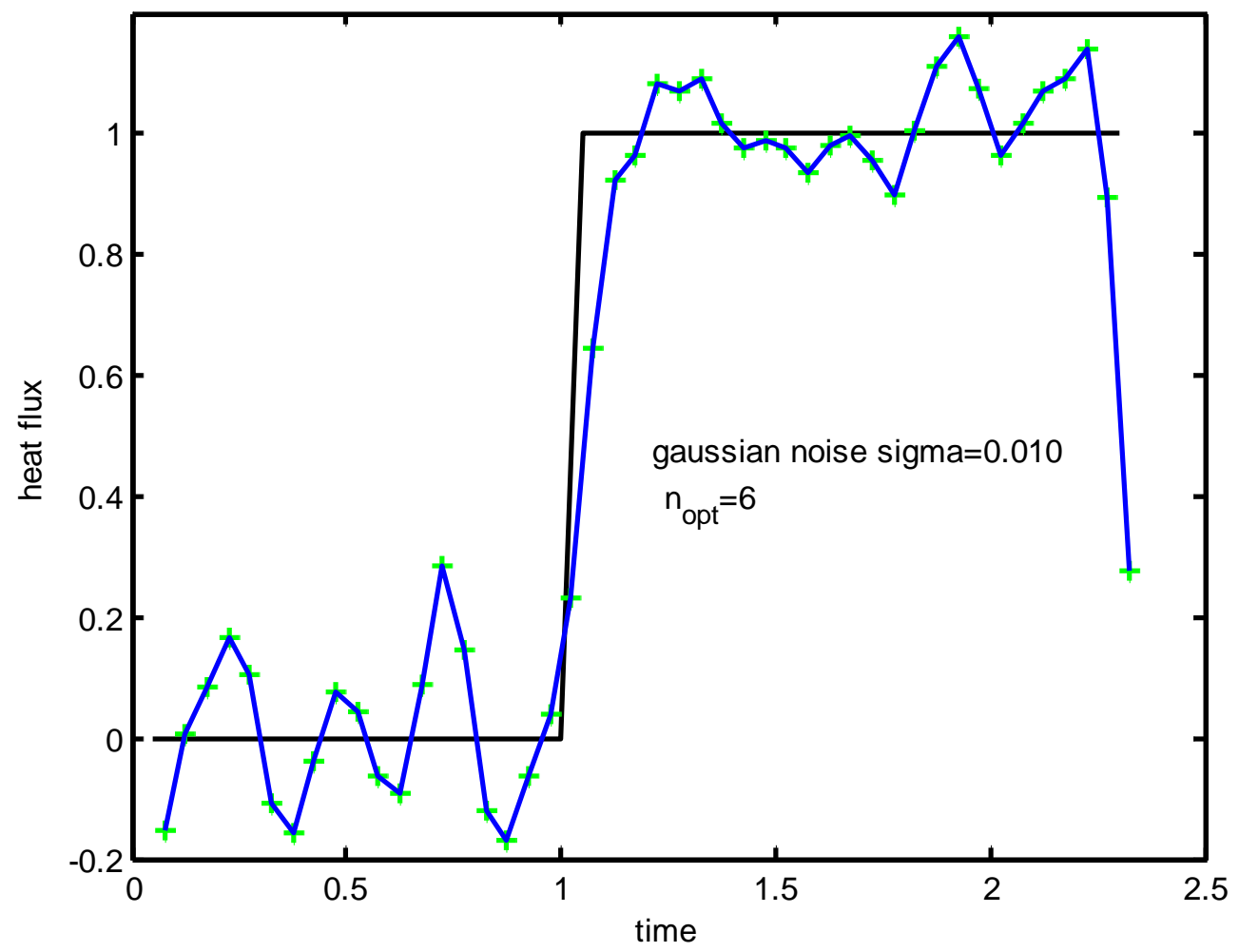

Fig. 12. Heat flux estimates for step heat flux obtained using CG method (Fletcher-Reeves algorithm). $\alpha \Delta t / L^{2}=0.05$, sensor at $x / L=0.5$; The sum of the heat flux errors squared $e_{q}^{2}=1.123$ 


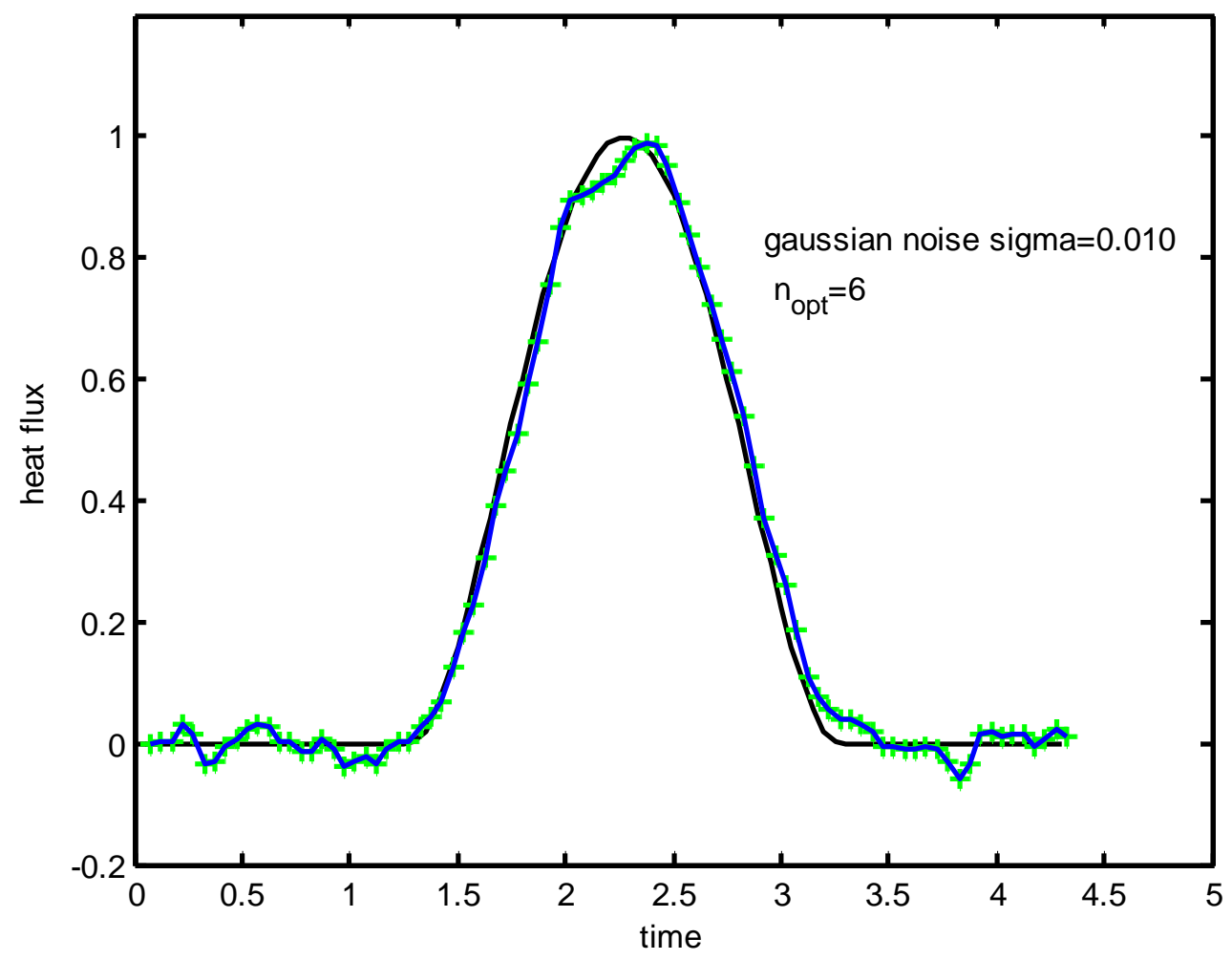

Fig. 13. Heat flux estimates for quartic heat flux obtained using CG method (Fletcher-Reeves algorithm). $\alpha \Delta t / L^{2}=0.05$, sensor at $x / L=0.5$; The sum of the heat flux errors squared $e_{q}^{2}=0.0444$ 


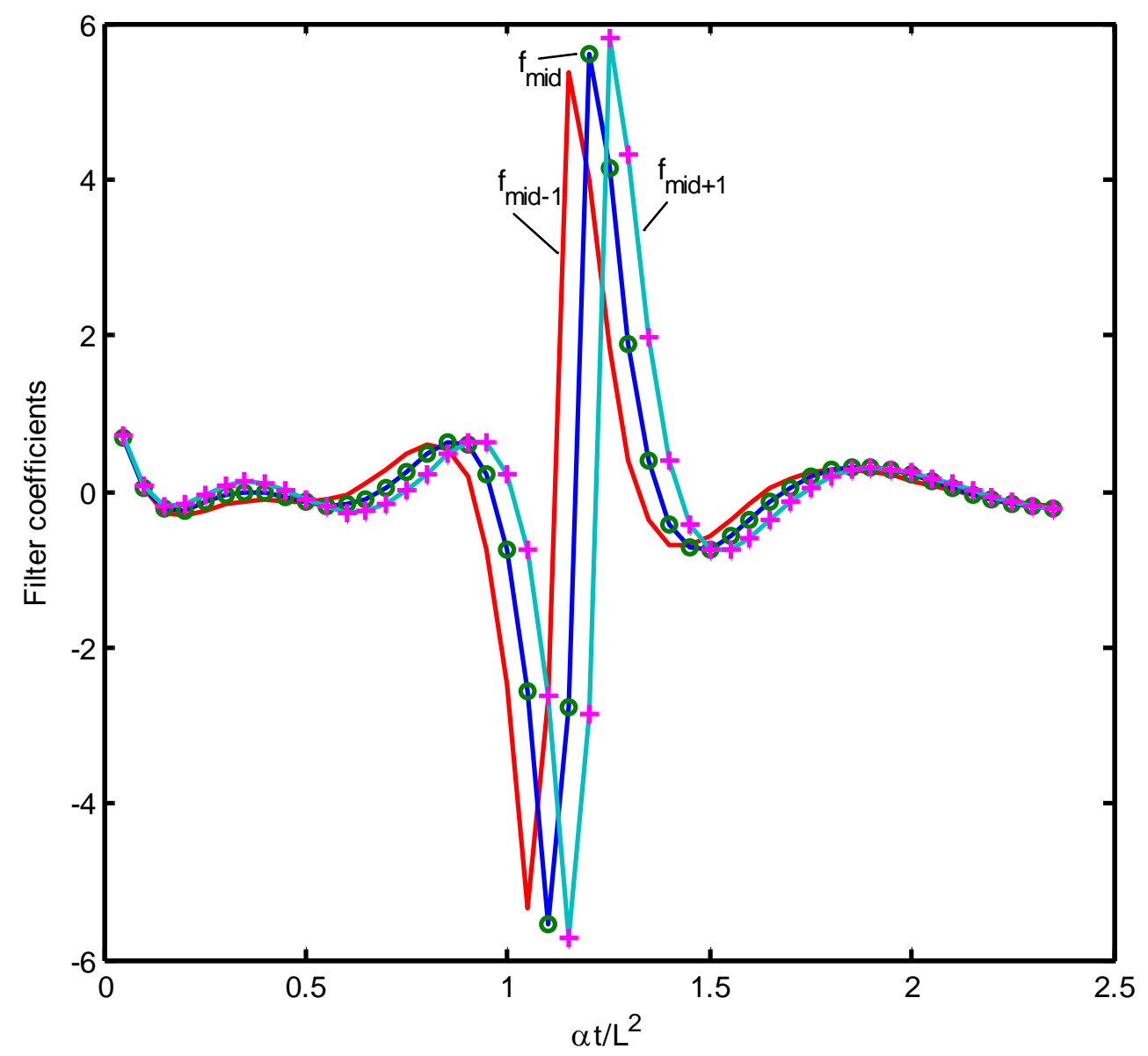

Fig. 14. Filter coefficients for the mid column and plus and minus 1 adjacent ones for the CGM (Fletcher-Reeves algorithm). $\alpha \Delta t / L^{2}=0.05$, sensor at $x / L=0.5$. GradientX22_2mat4.m. 


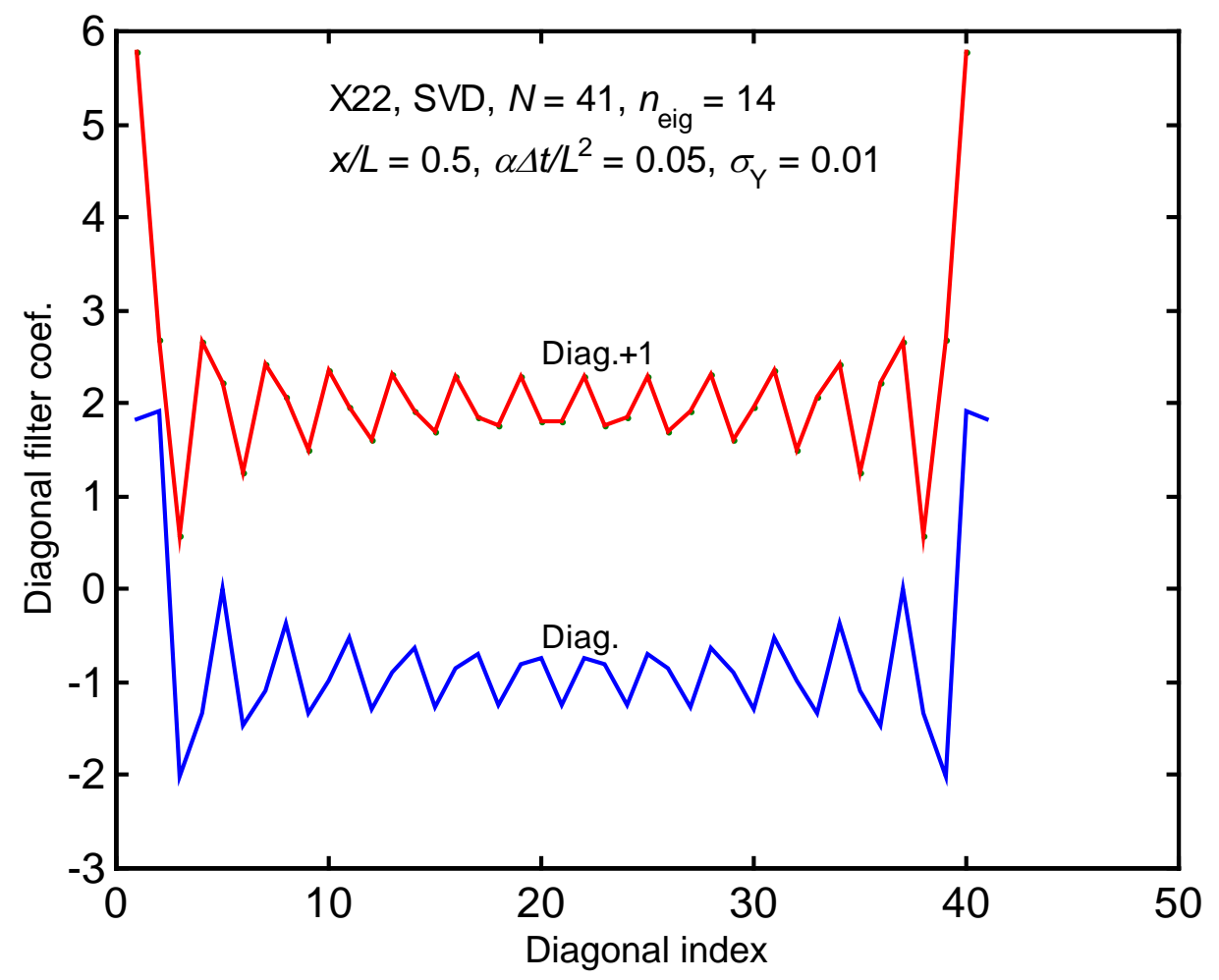

Fig. 15. Values of the filter along the main diagonal and diagonal plus 1 filter coefficients for

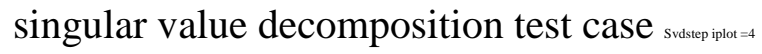




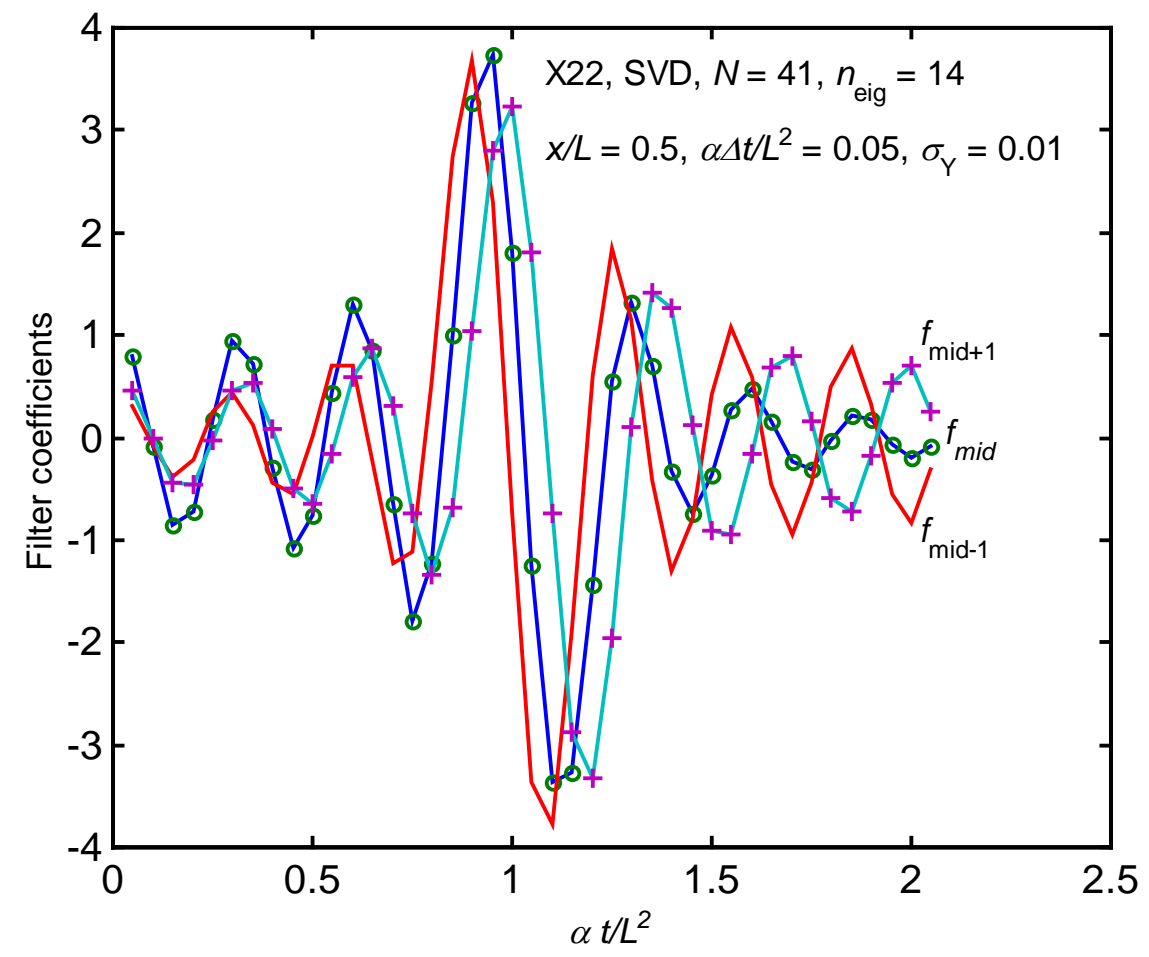

Fig. 16. Filter coefficients for the mid column and plus and minus 1 adjacent ones for the SVD case. Due to persymmetry, these column values are the same as corresponding rows. Svdstep iplot $=6$ 


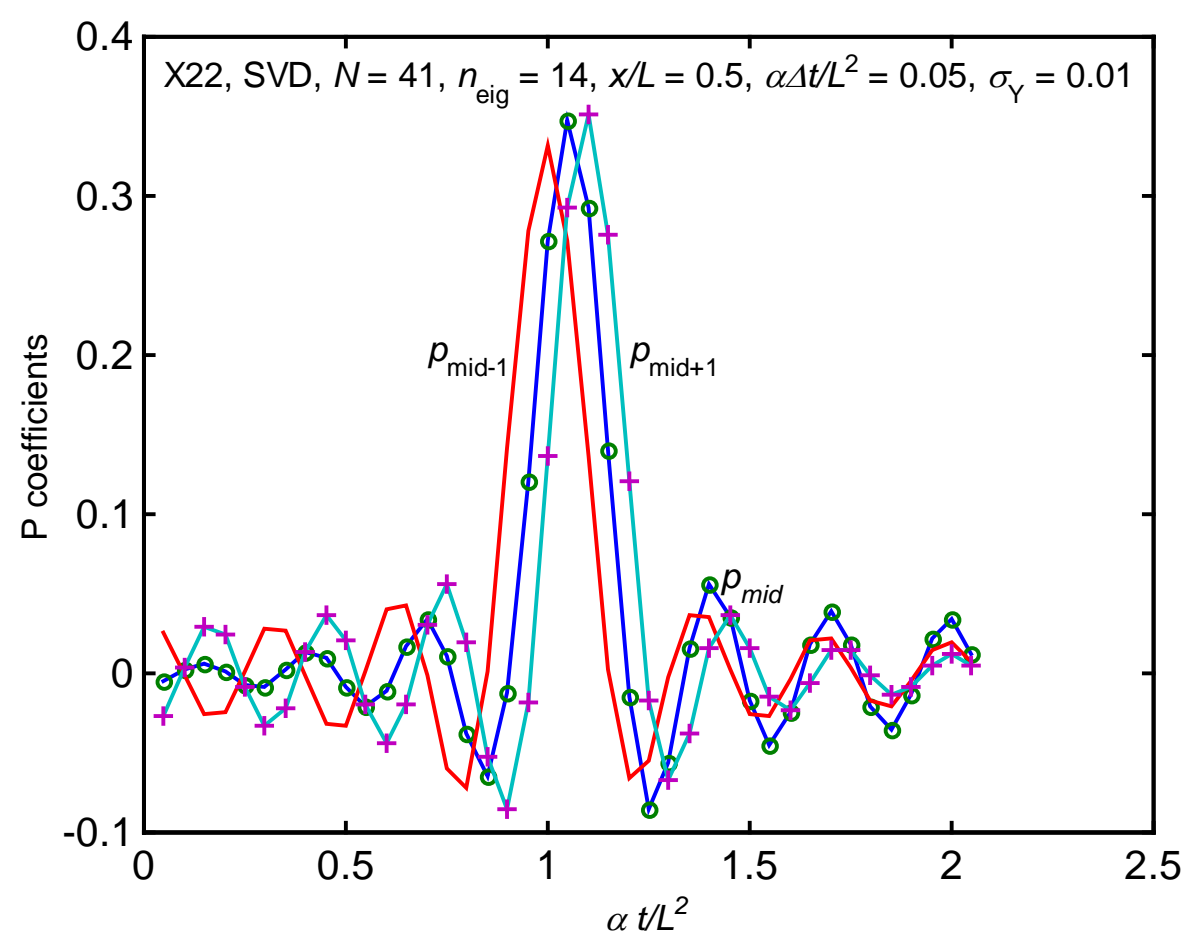

Fig. 17. $p$-coefficients for the mid column and plus and minus one adjacent columns for the SVD case. Svdstep iplot $=7$. 


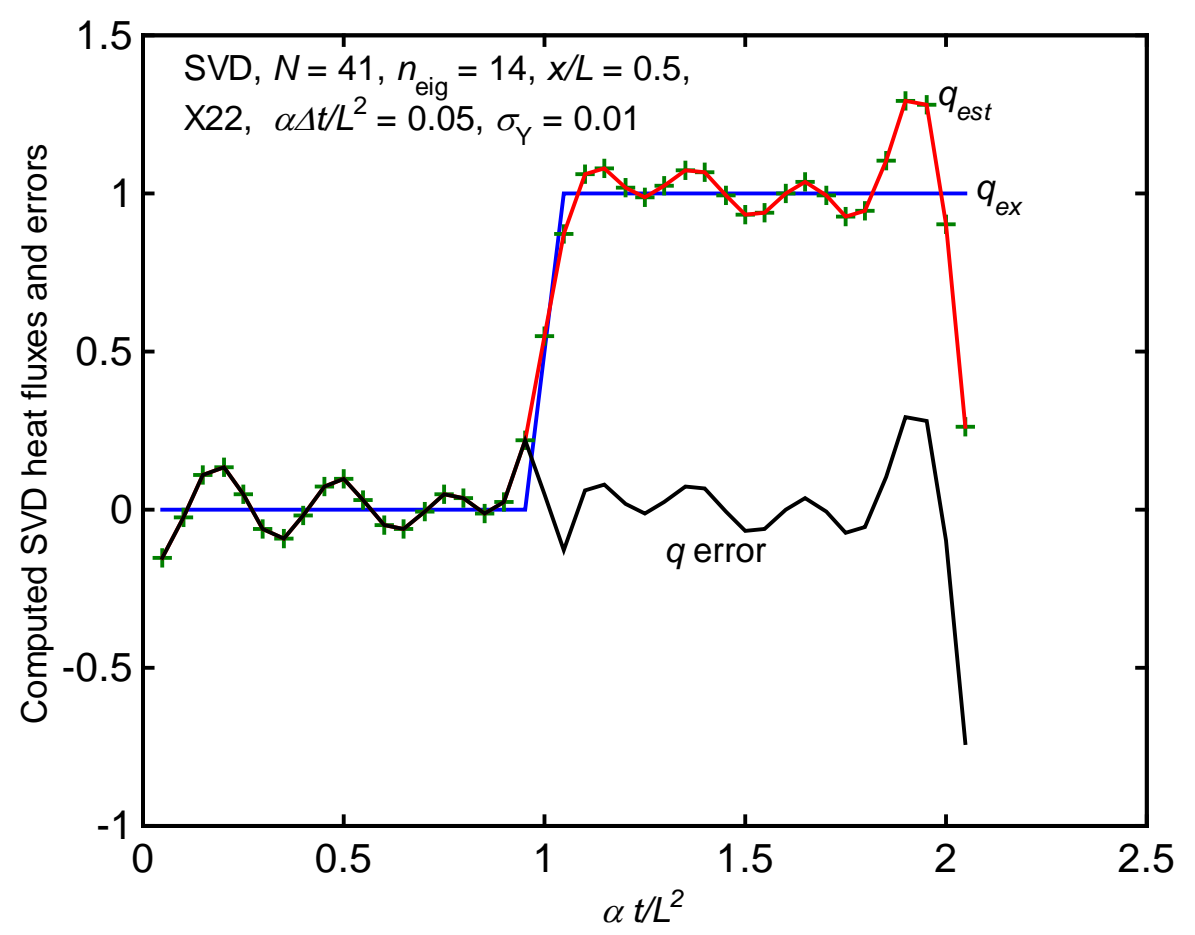

Fig. 18. Heat flux and errors for step heat flux using SVD with neig $=14$ and $\sigma_{Y}=0.01$. 


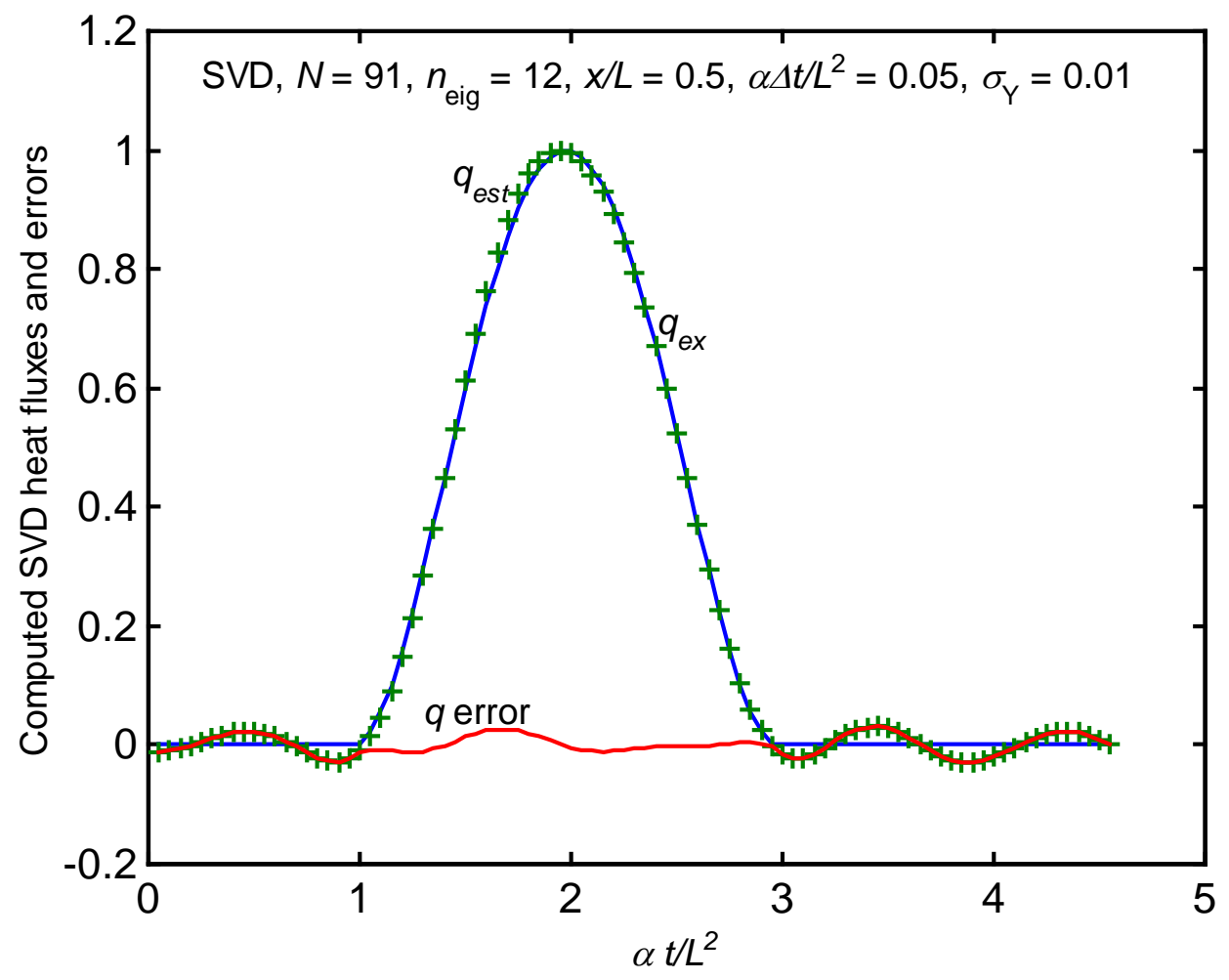

Fig. 19. Heat fluxes and errors for quartic heat flux using SVD with $n_{\text {eig }}=12, \sigma_{Y}=0.01$. Svdxtxaquartic iplot $=0$. 